\title{
2D-enabled membranes: materials and beyond
}

Taehoon Hyun ${ }^{1}$, Jinhong Jeong ${ }^{1}$, Ari Chae ${ }^{1,2}$, Young Kwan Kim² ${ }^{2^{*}}$ and Dong-Yeun Koh ${ }^{1,3^{*}}$ (D)

\begin{abstract}
Membranes could reform the field of molecular separations by enabling new low energy manufacturing technologies. This review article discusses the current state of the art and the potential in the 2D-enabled membrane separation processes by highlighting emerging and existing areas in which robust 2D materials significantly impact the energy-efficient separation process. Analysis of 2D-enabled membrane classes and prospective materials for 2D-enabled membranes are also discussed with emphasis on the surface chemistry of basal plane engineered 2D materials.
\end{abstract}

Keywords: Two-dimensional material, Membrane based separation process, Membrane fabrication

\section{Background}

Molecular separation processes are a critical compartment of the world's manufacturing infrastructure. In a current carbon-constrained world, separation processes present both challenges and opportunities for the development of low-energy, low-carbon manufacturing platform. Traditional thermal separation processes involving energetically less-favorable phase change but efficiently purify hydrocarbon streams still comprise up to $80 \%$ of current industrial separation platforms [1]. However, in seawater desalination, a non-thermal technique of membrane-based reverse osmosis (RO) has been a practical solution since the 1970s and currently dominates the worldwide market [2]. The drive behind this paradigm shift was simple-the operating energy cost for the pressure-driven $\mathrm{RO}$ process was roughly 5-10 times lower than thermal separation processes such as multi-stage flash (MSF) [3], and this revolutionary path represents the potential of membrane-based processes in diverse industrial areas. Industrial-scale membrane-based separation processes heavily rely on the development of more rigid polymeric barriers that can discriminate molecules with

\footnotetext{
* Correspondence: youngkwan@kist.re.kr; dongyeunkoh@kaist.ac.kr

${ }^{2}$ Carbon Composite Materials Research Center, Institute of Advanced

Composites Materials, Korea Institute of Science and Technology, Wanju-gun, Jeollabuk-do 565-905, South Korea

${ }^{1}$ Department of Chemical and Biomolecular Engineering (BK-21 Plus), Korea Advanced Institute of Science and Technology (KAIST), Daejeon 34141, South Korea

Full list of author information is available at the end of the article
}

very small size differences at high permeability [4]. Among newly emerging membrane materials with refined selectivities and permeabilities, materials fabricated in low-dimensional (preferentially in two-dimensional structures) architectures have the potential to combine excellent molecular specificity and flux when integrated into the membrane structure. Performance of the separation membranes, which exploit the effect of mass transport across a selective diffusion barrier to separate molecules, can be improved by reducing the thickness of the membrane. Ultimately achievable refinement in the thickness of materials is a one-atom-thick layer that can solely be achieved by $2 \mathrm{D}$ materials. Recently, ultrathin membranes have been fabricated using various techniques such as interfacial polymerization [5] (polyamide membrane $<10$ $\mathrm{nm}$ ), atomic-layer deposition ( $\mathrm{ZnO}$ layer thickness $\sim 200$ nm, effective ZIF-8 membrane would also be $<200 \mathrm{~nm}$ ) [6], and $3 \mathrm{D}$ printing (polyamide membrane $<20-80 \mathrm{~nm}$ ) [7]. In this review, we discuss 2D materials as building blocks for membrane structure, highlight recent development of 2D-enabled membranes, and outline future directions of 2D-enabled membranes.

Indeed, similar to inorganic membranes, membranes fabricated using 2D materials with refined micropores can be used to differentiate even very similarly sized compounds. Several key industrial sectors-pharmaceutical manufacturing, biofuel production, semiconductor manufacturing, and petrochemical separation-have the potential to be positively impacted by the use of 
advanced membranes to complement and supplement existing separation needs [8]. The critical need for membrane scientists and engineers facing more challenging molecular mixtures is the development of more robust, thin, and selective membrane materials. Specifically, 2D materials with few-atom-thick layers with engineered intrinsic (in-plane) micropores can be used to separate similarly sized molecules at high flux. These intrinsic pores or basal planes have the possibility to be functionalized with various chemical moieties, further refining the molecular specificity of the membranes. This review illustrates the challenges in permeability and molecular selectivity of the 2D-enabled membranes with variations on themes. A very distinct pattern in the development of the 2D-enabled membranes will be discussed followed by emerging 2D-enabled membrane materials that can be used in membrane architecture. The "molecular separations" can further be tuned via the engineered basal plane of various 2D materials, including nanocomposites, and these are discussed in respective sections. Finally, critiques and perspectives will be given on scalable development of 2D-enabled membranes with emphasis on future directions in the emerging areas.

\section{A transformative path for 2D-enabled membrane development}

The recent development of the 2D-enabled membrane architecture shows a distinctive pattern that differs from the development of the conventional polymeric membrane designs. Up to this point, three well-defined classes of the membrane systems have emerged with the development of 2D materials. Each class of the 2D-enabled membranes can be specified by the presence of the microporosity of the 2D building blocks and stacking structure (Fig. 1a-c). Representative molecular separation performance (for $\mathrm{H}_{2} / \mathrm{CO}_{2}$ gas pair) of the 2D-enabled membranes is shown in Fig. 1d. The class 1 membranes are lamellar-type membranes that are composed of compactly interlocked 2D nanosheets. Nanosheets used in this class have no intrinsic (in-plane) microporosity. Representative materials used in class 1 membranes are graphene $(\mathrm{G})$ and graphene oxide (GO) nanosheets. Tortuous transport pathways are formed between nonporous nanosheets that allow size-specific permeation of molecules (Fig. 1a). Since these nanoscopic pathways are produced in between the sheets, we define these as an external microporosity of the class 1 membranes. Class 1 membranes have shown the ability to
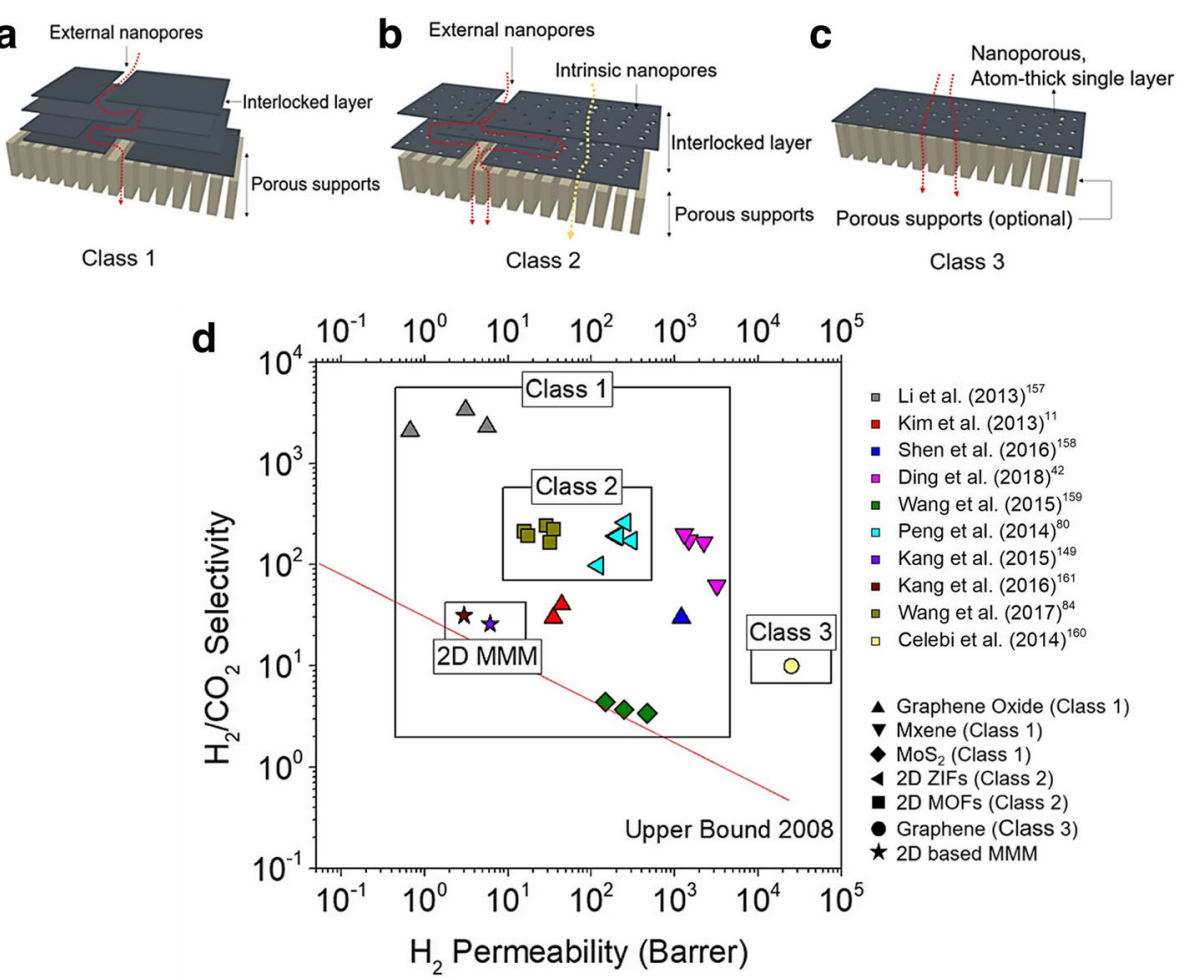

Fig. 1 Three classes of 2D-enabled membranes: a Class 1 membrane with highly interlocked 2D nanosheets. b Class 2 membrane with interlocked 2D nanosheets that have intrinsic microporosity. Red arrow represents transport pathways through the external nanopores and yellow arrow represents transport pathways through the intrinsic nanopores. c Class 3 membrane with one- or layered membrane. $\mathbf{d} \mathrm{H}_{2} / \mathrm{CO}_{2}$ separation performance (permeability and selectivity) of class 1 (graphene oxide [11, 157, 158], MXene [42], MoS 2 [159]), Class 2 (2D ZIFs [80], 2D MOFs [84]), Class 3 [160], and 2D nanosheet based MMM [149, 161]. Straight red line refers to 2008 upper bound [162] 
effectively remove solvated solutes from an aqueous feed at exceptionally high fluxes, as well as molecular size differentiation up to a $0.1 \mathrm{~nm}$ difference in kinetic diameter of the gas molecules. This suggests that the external microporosity spans the range from ultramicropore $(<$ $0.7 \mathrm{~nm})$ to supermicropore $(0.7-2.0 \mathrm{~nm})$ [9] based on different nanosheets and stacking modes.

Nair et al. [10] reported the permeation of water molecules through GO stacked membranes (class 1) with submicron thickness (Fig. 2a). The non-oxidized region of the GO laminate can form a nanoscopic capillary network for enhanced water transport in between GO sheets, while the oxide region provides mechanical support to maintain the laminate structure with specific interlayer spacing (Fig. 2b, c). Kim et al. [11] reported both few-layered graphene and graphene oxide membranes prepared in an interlocked structure. In the case of GO membranes, due to the affinitive interaction between a carboxylic acid group of GO and the effect of humidity, superior $\mathrm{CO}_{2}$ permeability and selectivity were observed. This class of the 2D membrane was further explored on a hollow fiber architecture by Huang et al. [12]. 2D membranes fabricated onto ceramic hollow fiber support showed excellent water permeation for various aqueous organic mixtures via a pervaporation process. Formation of the 2D-enabled membranes in a more scalable hollow fiber platform could open up the possibility of mass production of these newly emerging membrane architectures. For scalable manufacturing of these 2D-enabled membranes, roll-to-roll production of large area GO membranes also have been demonstrated [13]. Furthermore, various class 1 membranes based on reduced graphene oxide ( $\mathrm{rGO}$ ) membranes have recently been reported. Hung et al. [14] fabricated rGO membranes with a thickness of $50 \mathrm{~nm}$ with solvated rGO
(S-rGO). Nanoscopic channels produced in this way fell into the right size range for capably discriminating salt ions and dye molecules. Their membrane showed high water permeance of $80 \mathrm{Lm}^{-2} \mathrm{~h}^{-1} \mathrm{bar}^{-1}$ with a high rejection rate of Red 80 dye over $99.0 \%$. However, the exact separation mechanism (e.g., transport mechanism) of the graphene oxide membranes have not been clearly defined [15]. Since 2D-enabled membranes are in the early stage of development compared to other conventional membrane systems, several different analyses of the transport mechanism through the external micropores have been reported. Recently, Ibrahim and Lin proposed two separate transport mechanisms for GO-based membranes that can be categorized as an inter-sheet pathway (external micropore) and inner-sheet pathway (defective pathway) (Fig. 3). The authors suggested the inconsistency in reported permeabilities of GO-based membranes were due to the presence of the inner-sheet pathway, which is highly correlated with the defective sites of GO sheets. With their transport model, gas permeation through GO-based membranes can be categorized as Knudsen-like transport (inter-sheet pathway with a longer path length) and viscous transport (inner-sheet pathway with a shorter path length). Large gas molecules such as $\mathrm{CH}_{4}, \mathrm{~N}_{2}$, and $\mathrm{CO}_{2}$ are primarily transported through the inter-sheet pathway and smaller molecules like $\mathrm{H}_{2}$ and $\mathrm{He}$ permeates through either the inner- or inter-sheet pathway, which have a higher permeability for smaller gas molecules. For further development of class 1 (2D) membranes, the exact mechanism of gas permeations characterized by sorptivity, diffusivity, porosity and tortuosity of the nanosheets should be considered.

Conventionally, these class 1 membranes are fabricated using several different techniques such as vacuum
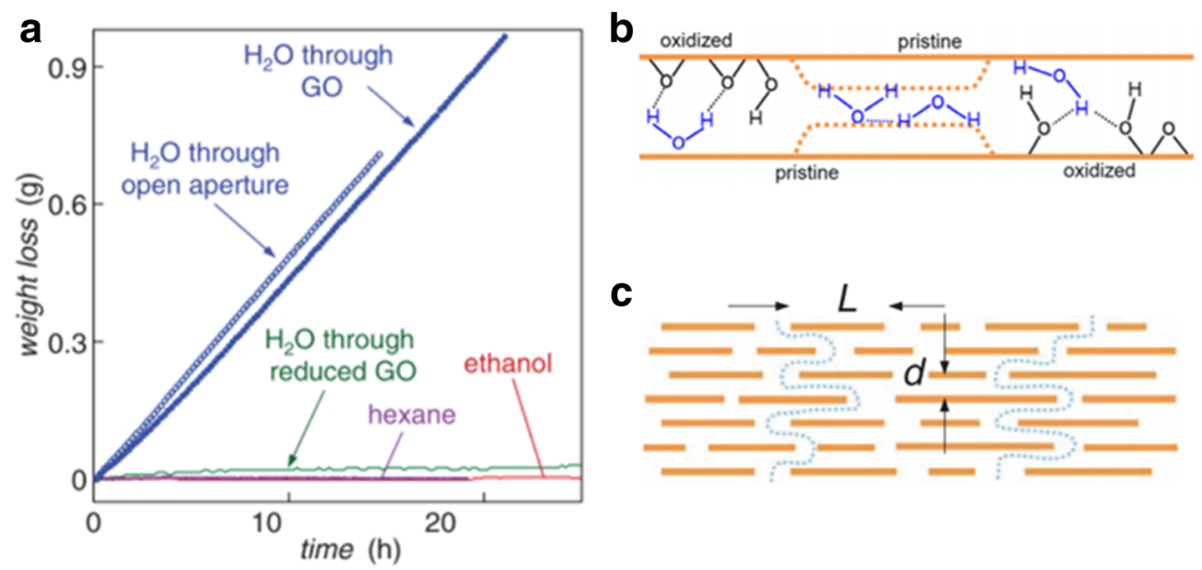

Fig. 2 a Permeation through the GO membranes. Water is freely evaporated through GO membrane similar to open aperture (blue line). b Structure of the single graphene capillary in the GO films. Pristine graphene capillaries form monolayer water channel. The dashed lines indicate the capillary state with low humidity. c Possible permeation routes for permeation through laminates. Adapted with permission from ref [10]. Copyright 2012 American Association for the Advancement of Science 


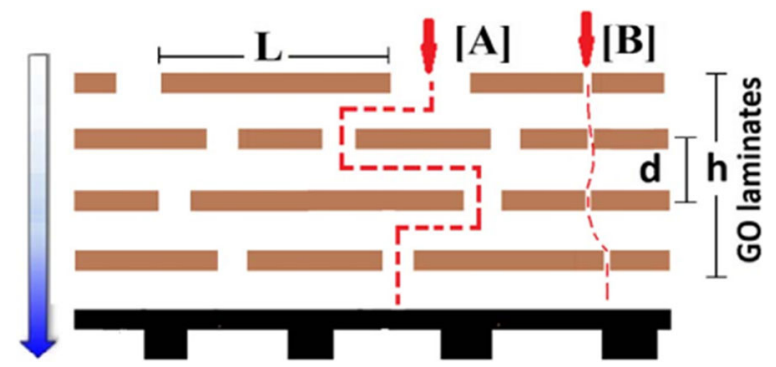

Fig. 3 Predicted transport model through GO membranes [A] path denotes the pathway through external nanopores and interlayer channel. [B] path denotes the pathway through the intrinsic pores of each layer [15]. Adapted with permission from ref [15]. Copyright 2018 for the Elsevier

filtration, vacuum suction, spray coating, spin coating, and dip coating. The most common method is dynamic vacuum filtration of the composite suspensions on a porous support to construct layer-by-layer deposited membrane. In this method, a solution containing well-dispersed 2D nanosheets are slowly filtered onto a desired porous support. Closely packed nanosheets are obtained after filtration that can be readily used in molecular separation testing. Even though the well-defined layered structure is achievable through filtration, it generally requires sufficient filtration time (up to several days) and constant driving force (vacuum level) that hinders rapid scale-up of this method. GO membrane prepared on a ceramic hollow fiber [12] is a good example showing the feasibility of this method. In this case, the vacuum filtration was performed on a ceramic hollow fiber with a sealed end. It was shown that uniform 2D membrane can be prepared on support with high curvature and elongated surface. In addition, various coating techniques are also applied to form 2D-enabled membranes. In "spray coating" method [16], dilute composites suspension with volatile solvents are sprayed onto the support. During the evaporation of solvents, 2D nanosheets are self-assembled into the lamellar-like structure. One advantageous feature of the spray coating method is that it can achieve ultrathin membranes by controlling the viscosity of the solution and spray pressure. In the case of "spin coating" method, the composite suspension is poured onto the center of the support and the suspension spread out via centrifugal force. Through this "spin coating" method [11], very thin, highly uniform and highly interlocked membranes based on 2D nanosheets can be formed. In "dip coating" method [17], the support is directly dipped into the composite suspension and the drawing out process forms a thin layer on the outer surface of the support. Similar to other coating methods, the 2D structure can be formed via evaporation and self-assembly.
Class 2 membranes are classified as 2D membranes that utilize nanosheets with intrinsic (in-plane) microporosity on the basal plane. Nanosheets with intrinsic microporosity capable of differentiating molecular size differences up to $0.01-0.05 \mathrm{~nm}$ are exploited in this class of membranes [18-20]. This intrinsic microporosity is different from external microporosity of the class 1 membranes and also differentiates itself from the intrinsic defects formed in 2D materials. Nanosheets derived (or delaminated) from 3D microporous crystalline materials such as zeolite [18], metal-organic framework (MOF) [19] and, covalent-organic framework (COF) [20] are a representative building block in class 2 membranes as well as "nanoporous" graphene or graphene oxide. When these ultra-microporous building blocks are efficiently packed into the lamellar structure, forming external microporosity via sheet stacking, class 2 membranes gain the potential to combine high molecular selectivity and flux stems from intrinsic micropores and external micropores. Since the discovery of exfoliated zeolite nanosheets, there have been many attempts to fabricate membranes with zeolite nanosheets. Zhang et al. [21] fabricated class 2 membranes with MFI zeolite nanosheets by a simple vacuum filtration method on a polymeric support. 2D Zeolite membranes produced in this way showed excellent $n$-butane permeance (up to 1000 GPU) with $n$-butane/iso-butane selectivity of $\sim 5$. Interlayers between zeolite nanosheets act both as a gallery for enhanced molecular transport and as size-selective transport pathways. Zeolite nanosheets have been used as seeds for further zeolite membrane growth-the hydrothermal secondary membrane growth efficiently closes the interlayer gaps and significantly enhances the membrane performances [22-24]. However, these secondarily grown membranes (inter-grown) are not in the scope of this review article. Nanoporous graphene or graphene oxide can be produced via etching of carbon atoms on a basal plane, which can form a pore with an area of $2.6 \AA^{2}$ per single carbon atom etched [25]. Weiwei et al. [26] used an oxygen plasma etching method to enhance the water permeation through a reduced graphene oxide ( $\mathrm{r}-\mathrm{GO}$ ) membrane by modifying the hydrophilicity of the surface of r-GO layers. Triple-layered graphene oxide membranes were formed by thermal reduction with exposure to oxygen plasma for 8-30 s. After the plasma treatment, water permeance through the r-GO membrane has significantly increased up to 4$40 \mathrm{Lm}^{-2} \mathrm{~h}^{-1}$ bar $^{-1}$ from less than $2 \mathrm{Lm}^{-2} \mathrm{~h}^{-1} \mathrm{bar}^{-1}$ of the non-treated $\mathrm{r}-\mathrm{GO}$ membrane. Formation of nanoporous layers can dramatically improve the membrane performance by forming a shortened transport pathway (Fig. 1b, Class 2) using in-plane nanoscopic pores-which is the main difference between class 2 membranes and class 1 membranes. Peng et al. [27] fabricated a 1-nm thick 
MOF nanosheet by a soft-physical exfoliation method using $\mathrm{Zn}_{2}$ (benzimidazole) ${ }_{4}$ as a parent 3D framework (Fig. 4a). Nanosheet suspension was drop coated on heated support (hot drop coating) that lead to disordered stacking due to the variation on the rate of evaporation across the film. In contrast, conventional filtration method applies constant force across the film, ordered restacking of the nanosheets are obtained, which have been identified from $x$-ray diffraction. However, the ordered stacking of MOF nanosheets showed partially or blocked micropores, therefore hot drop coating method was preferred to efficiently fabricate class 2 membranes for these materials. Their membranes showed impressive selectivity for the $\mathrm{H}_{2} / \mathrm{CO}_{2}$ pair $(\sim 260)$ at $120^{\circ} \mathrm{C}$ with linearly increased $\mathrm{H}_{2}$ permeance through the membranes at high temperature (Fig. 4b).

The ultimate class of the 2D-enabled membranes is the class 3 membranes that are one- or a few atoms thick layers with intrinsic microporosity. Class 3 membranes represent the lower limits for any membrane fabrication (one atom thick) combined with ultra-permeability via an atomically-thick membrane layer. Membranes in this class are a version extended from class 2 membranes by the formation of large-area atomically thin membranes. Generally, class 2 membranes have a wide range of thicknesses (a few tens of nanometers to a few micrometers) due to the stacking of nanosheets; however, class 3 membranes are classified as few nanometer thick membranes fabricated from 2D nanosheets. Single or few-atomic-layer membranes with tuned intrinsic micropores, such as graphene nanosheets with engineered nanoscopic pores are representative membranes in this class. The advantage of atomically thin membranes is their maximized molecular flux (minimized transport resistance) [28]. Although the graphene itself can form perfectly two-dimensional single atomic layers, a perfect single layer still cannot permeate a small gas such as helium. The molecular sieving potential of nanoporous graphene is gained from the fact that by selectively (and regularly) removing carbon atoms from the lattice, it should be possible to develop membranes that offer exquisite molecular sieving properties and ultra-high molecular fluxes. The membrane reported in Surwade et al. $[25,29]$ is one of the representative class 3 membranes. Surwade et al. created a one-atom-thick nanoporous graphene membrane using plasma etching. Single layer graphene was deposited on a copper surface and transferred to a silicon nitride wafer with $5-\mu \mathrm{m}$ holes. A short burst of oxygen plasma created nanoscopic pores on the graphene layer with sufficient areal density to allow ultra-high water flux combined with a nearly perfect salt rejection. Huang et al. [30] reported a relatively large area $\left(1 \mathrm{~mm}^{2}\right)$ single-layer graphene film as a class 3 gas separation membrane. To minimize the formation of cracks during transfer process from chemical-vapor deposition (CVD) derived graphene on porous supported graphene (Fig. 5a), researchers used nanoporous carbon as a sacrificial layer to protect the graphene membrane layer during the transfer process. Intrinsic defects of graphene that originated from the CVD process produced reasonably high hydrogen permeance (up to $4 \times 10^{-7} \mathrm{~mol} \mathrm{~m}^{-2} \mathrm{~s}^{-1} \mathrm{~Pa}^{-1}$ ) (Fig. 5b) with $\mathrm{H}_{2} / \mathrm{CH}_{4}$ selectivity up to 25 (Fig. $5 \mathrm{c}$ ), which was comparable to state-of-the-art polymeric membranes with $1 \mu \mathrm{m}$ thickness. Researchers further demonstrated ozone functionalization that enhanced the areal density of the nanoscopic pores on the basal plane of the graphene membrane, which is directly related to the productivity (flux) of the graphene-based membrane. One important question about class 3 membranes is the durability of


Fig. 4 a $A$ cartoon illustrating the gas separation through porous $Z n_{2}(B i m)_{3}$ nanosheets. Only $Z n$ atoms are shown for clarity, and the light blue planes represent the nanosheets regardless of their amphiprotic natures. The blue line indicates the pathway of $\mathrm{H}_{2}$ and the red line represents the pathway of $\mathrm{CO}_{2}$ [27]. $\mathbf{b}$ The change of permeance of $\mathrm{H}_{2} / \mathrm{CO}_{2}$ and mixture separation factor of $\mathrm{Zn}_{2}(\mathrm{Bim})_{3}$ nanosheet membrane for different temperature condition [27]. Adapted with permission from ref [27]. Copyright 2017 for the John Wiley and Sons 


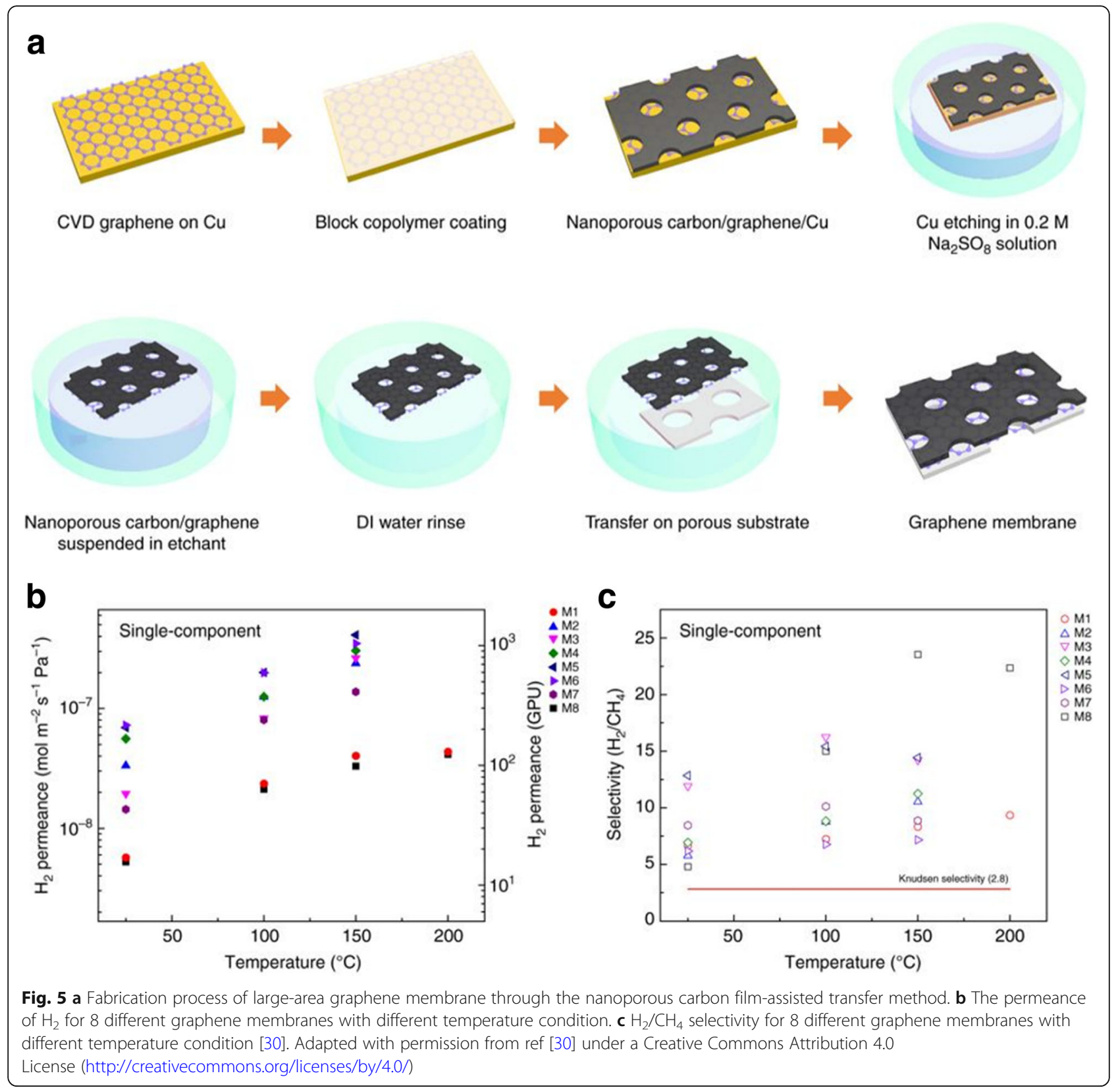

the single layer membranes at various pressure and temperature conditions. Kumar et al. [31] tested the stability of single-layer graphene membranes $(5 \mu \mathrm{m}$ diameter) by a temperature swing cycle $\left(25-200^{\circ} \mathrm{C}\right)$ with transmembrane pressure up to $0.5 \mathrm{bar}$. In various conditions, non-porous (as synthesized) single-layer graphene membranes showed impressive stability for several days. Then, researchers introduced an oxygen etching technique to create nanoscopic pores on the graphene layer to allow the transport of gas molecules. Although the pressure testing was not entirely investigated on the nanoporous graphene membrane, this result provided a guide for standardization of membrane testing procedures for $2 \mathrm{D}$-enabled membranes. Nevertheless, a fundamental understanding of the permselectivity of the class 3 membrane is still under development. A few mechanisms for gas permeation through the sub-nanometer graphene pores was proposed by Yuan et al. [32]. In their model, gas permeation through the graphene pores was described in a two-step process of adsorption and translocation. First, the gas molecules are adsorbed on the entrance of the generated pores and these are translocated along the graphene membrane via a chemical potential gradient. Then the rate coefficient of translocation obeys Arrhenius-type behavior, indicating that the 'solution-diffusion model' defined in 
conventional membrane systems with a continuum-level assumption might be applied to the single-layer 2D membranes. The transport models of 2D-enabled membranes are suggested by several reports. Graphene based class 3 membranes are often treated as a test bed for modeling work on atomically thin membranes. For class 3 membranes, the relative size of pores and the penetrating molecule is a dominant factor that can ultimately modify the permeability through the membrane [33, 34]. For relatively large pores (pore size larger than the penetrants), steric regime model can be applied to describe the transport of gas molecules [35]. In this model, gas molecules are treated as a rigid sphere with specific kinetic diameters. Steric regime model suggests no interaction between the pore edge sites and penetrants, therefore energetic barrier for gas transport in the pore is low. On the other hand, activated regime model assumes that certain energy barrier exists when the penetrants attempt to pass through the pores. In this case, Lennard-Jones potential is used to describe penetrant-pore interaction [36]. For relatively small pores (pore size smaller than the penetrants), activated regime model is used to predict molecular transport across the membrane. Limitations of these models include lack of consideration of the adsorptive flux near the nanosheet surface and the detailed chemical structure of nanopores. Du et al. [37] and Sun et al. [38] reported the significance of adsorptive flux near the surface of graphene through molecular simulations. The affinity difference between gas molecules with a carbon atom of the graphene layer will alter the permeability across the class 3 graphene membrane and eventually affect the selectivity of different gas pairs. For example, $\mathrm{N}_{2}$ can form the adsorptive flux near the graphene layer through van der Waals interaction that leads to high $\mathrm{N}_{2}$ / $\mathrm{H}_{2}$ selectivity. In summary, to consider the transport model in 2D-enabled membranes, in addition to the pore size, the interaction between the penetrants and 2D materials should be fully considered. Although research into the nanoporous graphene membrane (class 3 ) is still in its early stages, these membranes will open up the new possibilities in molecularly-selective separation processes for future generations.

\section{Newly emerging materials as "contenders" in 2D- enabled membranes}

The development of the 2D-enabled membranes to date have been largely led by the use of graphene and graphene oxide. Particularly, graphene oxide (GO) is prepared inexpensively on a large scale by oxidation and exfoliation of graphite. These GO nanosheets can be easily dispersed in water and provide an easy means for membrane fabrication via conventional filtration technique. On the laboratory scale, a $1-10 \mathrm{~cm}^{2}$ membrane sample is sufficient to generate transport data. However, the large-scale application requires membranes scaled up in a few orders of magnitudes in a defect-free manner [39]. Fabrication of defect-free, large-scale graphene or graphene oxide membranes for gas and liquid separation has not yet been fully exploited and is sometimes extremely challenging due to their poor mechanical properties. Moreover, forming uniform intrinsic micropores on the graphene layer (class 3 membranes) or tuning the uniform tortuous path (external micropores in class 1 or 2 membranes) in laminate graphene oxide membranes are still questionable [40]. In this section, we discuss the applicability of the recently emerging $2 \mathrm{D}$ nanosheets other than graphene-derived materials in a membrane structure that may help to advance the development of 2D-enabled membranes. More detailed surface chemistry and functionalization of the 2D materials will be discussed in the following separate section.

\section{MXenes}

MXenes are the inorganic compound that has a chemical formula of $M_{n+1} X_{n} T_{x}$ (M: Transition metal, X: Carbon or nitrogen, T: Functional group on the basal plane). MXenes are derived from the parent MAX phases, where A consists of $3 \mathrm{~A}$ metals or $4 \mathrm{~A}$ elements. In the MAX phases, M-A bonds have weaker interaction than $\mathrm{M}-\mathrm{X}$ bonds, so A element can be selectively removed at high temperature or etching by HF treatment. Removal of the $\mathrm{A}$ induces the formation of MXene nanosheets with a form of $\mathrm{M}_{2} \mathrm{X}, \mathrm{M}_{2} \mathrm{X}_{2}$, and $\mathrm{M}_{4} \mathrm{X}_{3}$ [41]. Representative MXene family members include $\mathrm{Ti}_{3} \mathrm{C}_{2}, \mathrm{Ti}_{2} \mathrm{C}, \mathrm{Nb}_{2} \mathrm{C}$, $\mathrm{V}_{2} \mathrm{C},\left(\mathrm{Ti}_{0.5} \mathrm{Nb}_{0.5}\right)_{2} \mathrm{C},\left(\mathrm{V}_{0.5} \mathrm{Cr}_{0.5}\right)_{3} \mathrm{C}_{2}, \mathrm{Ti}_{3} \mathrm{CN}$ and $\mathrm{Ta}_{4} \mathrm{C}_{3}$. In MXene nanosheets, the functional groups $(\mathrm{T})$ play a critical role in creating the nanochannels between two MXene nanosheets. Functional groups contribute to the stability of MXene membranes by adjusting the interaction between the individual MXene nanosheets. Using these MXene nanosheets, various class 1 membranes have been demonstrated in gas and liquid separations. Ding et al. [42] fabricated membranes with titanium-based MXenes $\left(\mathrm{Ti}_{3} \mathrm{C}_{2} \mathrm{~T}_{\mathrm{x}}\right)$ by vacuum filtration on an anodic aluminum oxide support. The interlayer spacing between MXene layers was $\sim 0.35 \mathrm{~nm}$ and allowed the high permeability of the gas molecules such as hydrogen (2100 Barrer) and He (2400 Barrer) (Fig. 6). Due to the much smaller interlayer spacing provided in MXene based membranes compared to GO based membranes, MXene based membranes show promising molecular sieving properties for the $\mathrm{H}_{2} / \mathrm{CO}_{2}$ pair with selectivity over 100. Chemical functionalization on the MXene surfaces can further refine the selectivity of specific gaseous species. Jie et al. [43] used borates to selectively transport $\mathrm{CO}_{2}$ through MXene channels by crosslinking the borates $\left(\mathrm{B}_{4} \mathrm{O}_{7}{ }^{2-}\right)$ with hydroxyl groups 


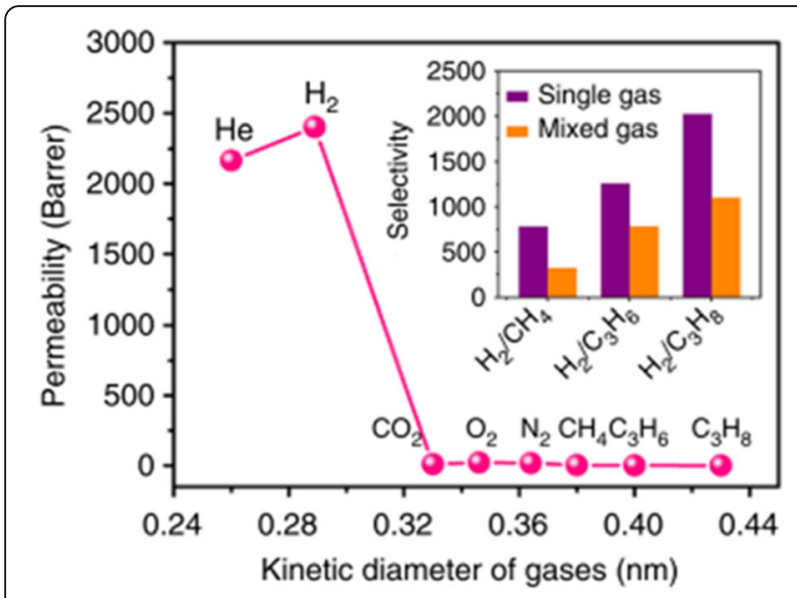

Fig. 6 Single-gas permeability of various gases with different kinetic diameters across the 2 - $\mu$ m-thick MXene membrane at $25^{\circ} \mathrm{C}$ and 1 bar. The inner graph showed the selectivity of 3 equimolar mixed gases [42]. Adapted with permission from ref [42]. Copyright 2018 for the John Wiley and Sons

on the surface of MXene nanosheets (Fig. 7a). It reduced the interlayer distance between MXene nanosheets, thus confirming the reversible desorption of trapped $\mathrm{CO}_{2}$ in the nanosheets. At $75^{\circ} \mathrm{C}$, the borate modified MXene membrane showed $13 \%$ enhancement of $\mathrm{CO}_{2}$ adsorption capacity compared to a pristine MXene membrane (Fig. 7b). Further interlocking between MXene nanosheets was achieved by cross-linking the borate and polyethyleneimine (PEI). Positively charged PEI molecules help to reduce the interlayer spacing when mixed with borate and MXene flakes, tuning the interlayer spacing down to $0.34 \mathrm{~nm}$ at $75^{\circ} \mathrm{C}$-which fall into the range of kinetic diameters of $\mathrm{CH}_{4}(0.36 \mathrm{~nm})$ and $\mathrm{CO}_{2}(0.32 \mathrm{~nm})$. MXene with borate and PEI achieved 5.2 times enhancement of $\mathrm{CO}_{2}$ permeance compared to the pristine MXene membrane (350 GPU for MXene with borate and PEI and 67 GPU for pristine MXene membrane).

MXene based membranes also show promising liquid separation performances. Chang et al. [44] reported MXene membranes that had ultrafast water flux and high ion selectivity. One interesting feature is that even with a relatively thick membrane $(0.9-1.5 \mu \mathrm{m})$, their membrane achieved very high water permeance $(\sim 38$ $\left.\mathrm{Lm}^{-2} \mathrm{~h}^{-1} \mathrm{bar}^{-1}\right)$, which was much higher than that of the $1.5 \mu \mathrm{m}$ thick GO membranes $\left(\sim 6.5 \mathrm{Lm}^{-2} \mathrm{~h}^{-1} \mathrm{bar}^{-1}\right)$. Researchers suggested that the thin layer of water molecules formed between the MXene nanosheets, which provided a fast transport pathway for water molecules, was responsible for this high water permeance. In addition, their membrane showed size and

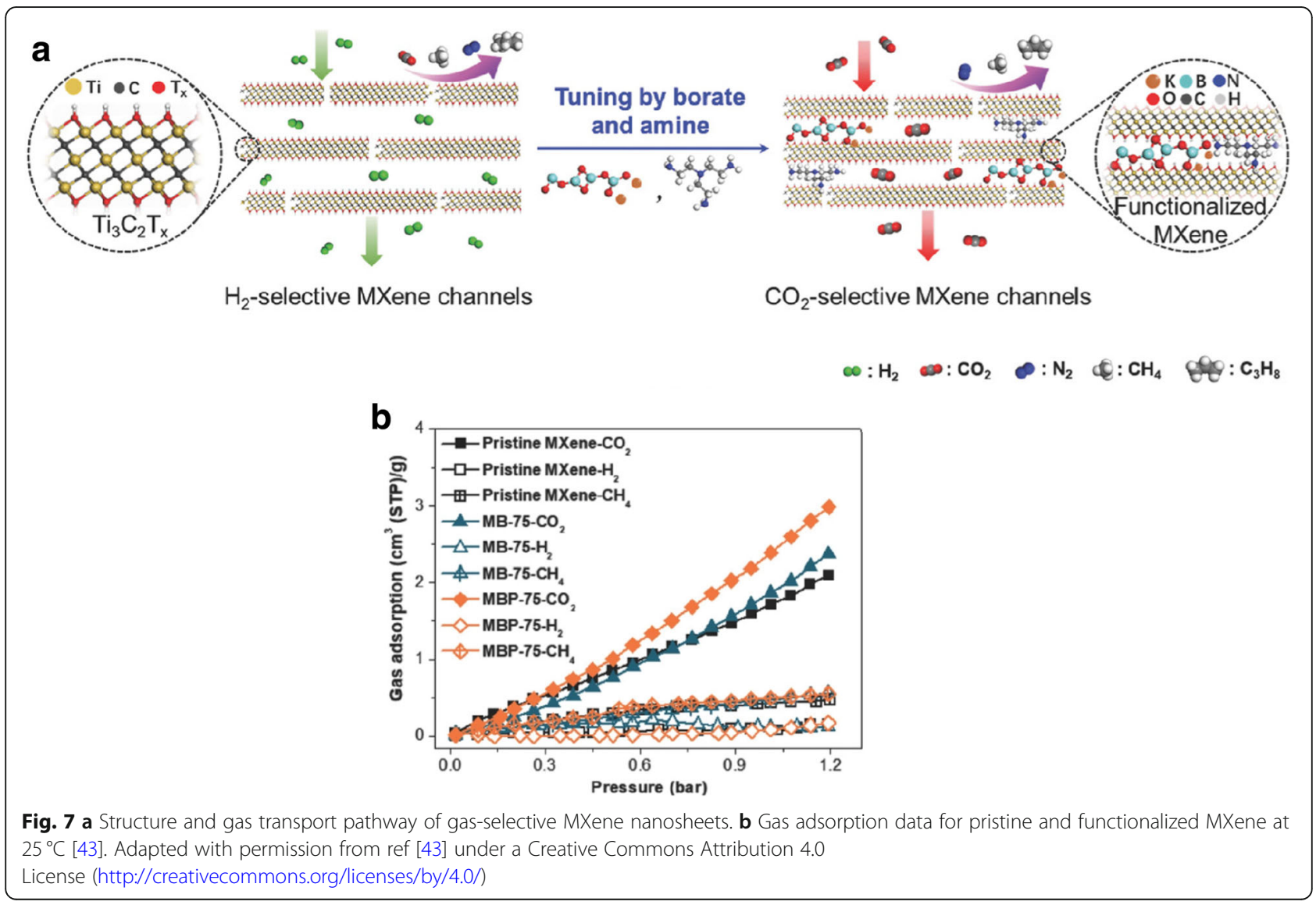


charge-selective permeation of ions. $\mathrm{Na}^{+}$had the highest permeation rate among differently charged cations, achieving a 25 times higher permeation rate compared to $\mathrm{Al}^{3+}$ and 7 times higher than $\mathrm{Ca}^{2+}$. The ionic selectivity of the MXene based membranes reflects that the MXene nanosheets are also promising building blocks for 2D-enabled membranes. Wang et al. [45] fabricated $4.5 \mathrm{~nm}$ thick lamellar MXene nanosheets that attained surprisingly high water permeances $\left(\sim 2300 \mathrm{Lm}^{-2} \mathrm{~h}^{-}\right.$ ${ }^{1} \mathrm{bar}^{-1}$ ) and organic solvent permeances (up to 5000 $\mathrm{Lm}^{-2} \mathrm{~h}^{-1} \mathrm{bar}^{-1}$ for both acetone and acetonitrile). These permeances are at least 20 times higher than those of the $18 \mathrm{~nm}$ thick reduced GO membranes for acetone. The regular and ordered flow channels created between MXene nanosheets were the vehicle for the high water and solvent permeances, opening up the possibility of the use of MXene membranes in the field of organic solvent nanofiltration (OSN). Li et al. [46] used a molecular dynamics simulation in a quest to find the optimal design of the MXene lamellar structure for selective molecular separations. Two factors, interlayer distance (d) and intercalated molecules were identified as critical factors in designing MXene based membranes for optimal separation performances. The interlayer distance (d), of course, provides tortuous pathways for molecular transport and plays a critical role for molecular sieving of similarly sized molecules. By simulating the variation in the interlayer distance from 5 to $13 \AA$, the authors showed that anhydrous MXene nanosheets with $\sim 5 \AA$ interlayer distance can effectively separate $\mathrm{H}_{2} / \mathrm{CO}_{2}$ (kinetic diameter $\mathrm{H}_{2}$ is $2.89 \AA$ and $\mathrm{CO}_{2}$ is $3.3 \AA$ ) mixture. In addition, the intercalated molecules between MXene sheets can modify the transport characteristics of the permeating molecules by partially tuning the "effective interlayer distance". Through the simulations, the diffusivity of several gas molecules in the hydrous (water-filled) MXene channel was calculated smaller than that of anhydrous MXene channels. When MXene sheets are hydrated, these water molecules can scatter the gas molecules or even block the pores, therefore decreasing the diffusion coefficient of gas molecules. For very small interlayer distance, water molecules even can block the gas transport channel of MXene nanosheets. The suggestions from their molecular simulation can be broadly used in most of the class 1 type membranes.

\section{Boron nitride (BN)}

Two dimensional boron nitride nanosheets are sometimes referred to as "white graphene" since they are the isomorphs of the graphene structure. 2D hexagonal boron nitride (h-BN) can be formed in a graphene-like structure by the mechanical cleavage from bulk BN crystal or the chemical vapor decomposition reactions of precursor molecules [47]. Its high mechanical and chemical stability is reported by multiple studies [4851]. Both graphene and BN shows hexagonal atomic structure formed with strong covalent $\mathrm{sp}^{2}$ bonds. However, $\mathrm{BN}$ exhibits intrinsically inorganic nature whereas graphene shows partially organic nature, therefore $\mathrm{BN}$ is generally treated as a chemically more inert compared to graphene. However, for fabricating a large area of h-BN nanosheets, thermal catalytic chemical vapor deposition is highly favored over the mechanical cleavage techniques, partially overcoming the scalability problems related to 2D materials [47]. Each h-BN nanosheet has a honeycomb structure consisting of a borazine ring $\left(\mathrm{B}_{3} \mathrm{~N}_{3} \mathrm{H}_{6}\right)$ with a B-N bond length of $1.45 \AA$. The center-to-center distance of the adjacent borazine rings is $2.50 \AA$, which is slightly longer than $2.46 \AA$ in the graphene basal plane [52]. In addition, the stack order of $\mathrm{h}-\mathrm{BN}$ is different from that of graphene. In the graphene layers, each carbon atom is located right above the center or the carbon ring ( $\mathrm{AB}$ stacking); however, in h-BN, each $\mathrm{B}$ atom eclipses an $\mathrm{N}$ atom on the adjacent $\mathrm{h}-\mathrm{BN}$ layer (AA' stacking). Sutter et al. [53] reported the chemical vapor deposition (CVD) method to fabricate large-area hexagonal boron nitride (h- $\mathrm{BN})$ monolayers. With the low partial pressure of borazine, h-BN sparsely nucleated the domain and merged into a single layer. They also reported that the amount of hydrogen gas in the precursor gas controlled the expansion and morphology of growing h-BN domains. In addition, hydrogen gas inhibited the formation of surface oxides on metal substrates. The additional $\mathrm{H}_{2}$ with the borazine precursor provides evidence that the $\mathrm{B}$ and $\mathrm{N}$ incorporation at the $\mathrm{h}-\mathrm{BN}$ edge can be modulated to control the growth and to achieve different types of h-BN domain morphology. Especially, unlike graphene derivatives, h-BN has high chemical stability under both acidic and basic conditions. Although boron nitride has high stability in harsh conditions, it is difficult to fabricate class 1 membranes with h-BN nanosheets due to its hydrophobicity, which highlights the importance of functionalization of $\mathrm{h}$-BN to enhance the stacking properties of $\mathrm{BN}$ based membranes. Chen et al. [54] reported the use of amino-functionalized h-BN membranes in organic solvent nanofiltration (OSN). Class 1 membranes were fabricated using water-dispersible (functionalized) BN nanosheets. Membranes formed using functionalized boron nitride nanosheets have a superior solvent flux while also having moderate-to-high solute rejections in both organic and aqueous solvents. Their $400 \mathrm{~nm}$-thick membranes showed a water flux of $1500 \mathrm{Lm}^{-2} \mathrm{~h}^{-1}$ and 99\% rejection rate for Congo Red dye $(696.7 \mathrm{~g} / \mathrm{mol})$. However the rejection rates for methylene blue (MB, $320 \mathrm{~g} / \mathrm{mol}$ ), Rhodamine B (RhB, $479 \mathrm{~g} / \mathrm{mol})$, and Rhodamine 6G (R6G, $479 \mathrm{~g} / \mathrm{mol}$ ) were $50.3,58.3,83.8 \%$, respectively. For ethanol, it showed an ethanol flux of 
$1480 \mathrm{Lm}^{-2} \mathrm{~h}^{-1}$ with $54 \%$ rejection rate for Congo Red dye and $620 \mathrm{Lm}^{-2} \mathrm{~h}^{-1}$ with $99 \%$ rejection rate for $\mathrm{Au}$ nanoparticles. And for methanol it showed the flux of 600 , 740, 640 and $560 \mathrm{Lm}^{-2} \mathrm{~h}^{-1}$ with 99, 60, 88.7 and 99\% rejection rate for Congo Red dye, methylene blue (MB), Acid fuchsin (ACF, $586 \mathrm{~g} / \mathrm{mol}$ ), Evans blue (EB, $961 \mathrm{~g} / \mathrm{mol}$ ), respectively. In addition, compared to graphene derivatives, the functionalized inorganic boron nitride membrane demonstrated high stability for acid, alkali, and oxidative media conditions. Lei et al. [40] reported the adsorptive separation performance of $\mathrm{BN}$ laminates for oils, organic solvents, and water. The layered structure of the $\mathrm{BN}$ was synthesized via a dynamic templating approach, and $\mathrm{BN}$ laminate attained a notable BET surface area $\left(\sim 1400 \mathrm{~m}^{2} \mathrm{~g}^{-1}\right)$ and pore volume $\left(\sim 1 \mathrm{~cm}^{3} \mathrm{~g}^{-1}\right)$ indicating the possible application of the BN lamellar structure in membrane-based separations. Liu et al. [55] fabricated a mixed-matrix membrane (MMM) of poly (vinylidene fluoride) and boron nitride nanosheets.
Mixed-matrix membranes (MMMs) formed with 2D materials is further discussed in the following section, so it would be appropriate to briefly introduce the MMM formed with $\mathrm{BN}$ nanosheets here. Their membrane had a thickness of around $300 \mu \mathrm{m}$ and the BN nanosheets had many holes with a diameter range from 20 to $100 \mathrm{~nm}$. A $300 \mathrm{~mm}$ thick MMM fabricated with BN nanosheets achieved largely enhanced water permeability $\left(\sim 8.2 \times 10^{4}\right.$ $\left.L-\mu \mathrm{m}-\mathrm{m}^{-2} \mathrm{~h}^{-1} \mathrm{bar}^{-1}\right)$ that was three orders of magnitude higher than that of the pristine polyvinylidene fluoride (PVDF) membranes. Moreover, their membrane showed excellent filtration efficiency for several pharmaceuticals, such as carbamazepine, indicating the applications in organic solvent nanofiltrations.

\section{Molybdenum disulfide $\left(\mathrm{MoS}_{2}\right)$}

More than 60 types of transition metal dichalcogenide (TMD) have been discovered since 1960, and

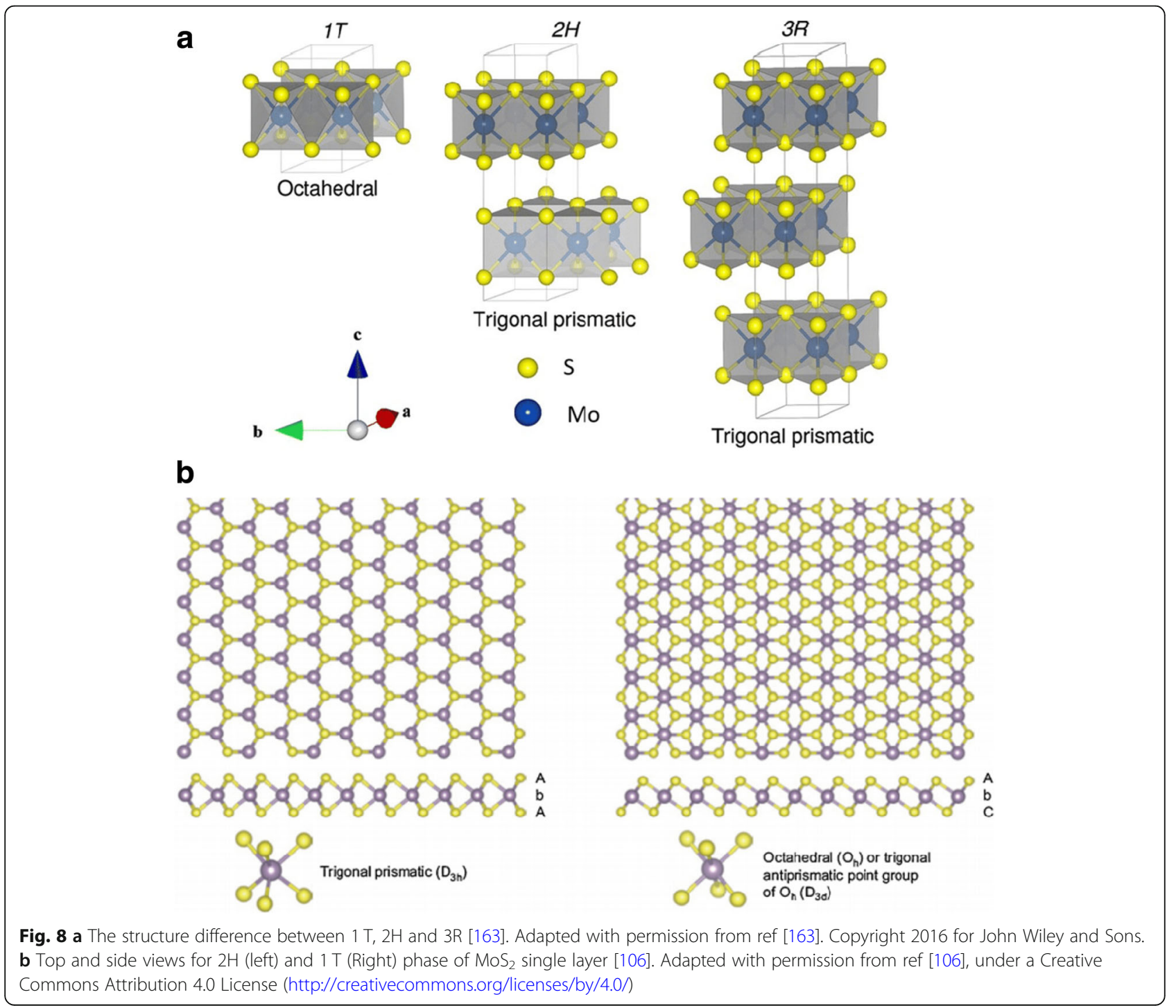


molybdenum disulfide $\left(\mathrm{MoS}_{2}\right)$ is one of the popular materials in this class and also a strong contender as building blocks for 2D-enabled membranes. Among various TMDs, 2D $\mathrm{MoS}_{2}$ is characterized by the well-defined, uniform two-dimensional transport channels with limited undesirable chemical defects [56]. The uniform nanoscopic channel structure of $\mathrm{MoS}_{2}$ is very advantageous for studying fundamental transport mechanisms in tortuous pathways in class 1 or class 2 membranes. In general, TMDs consist of a transition metal layer sandwiched between two chalcogen layers. Compared to other two-dimensional membrane materials, the TMD monolayer is unique for this "sandwiched structure" [57] (Fig. 8a). Due to this sandwiched structure, TMDs can have different crystal polytypes. For example, $\mathrm{MoS}_{2}$ can have four different crystal structures, $2 \mathrm{H}, 1 \mathrm{~T}, 1 \mathrm{~T}$ ' and $3 \mathrm{R}$ [58], with different coordination models. The $2 \mathrm{H}$ type has trigonal prismatic coordination consisting of hexagonally packed atoms with ABA staking mode (S-Mo-S'). The $1 \mathrm{~T}$ type has an octahedral phase. It has a stacking mode of $\mathrm{ABC}$ (S-Mo-S'). $1 \mathrm{~T}$ ' type is called 'distorted $1 \mathrm{~T}$ type'. It also has an octahedral phase, however, superlattice structures can exist in each layer. The $3 \mathrm{R}$ type also has trigonal prismatic coordination. The multilayers of 3R type showed a rhombohedral symmetry. Although $\mathrm{MoS}_{2}$ can have diverse crystal structures, due to the thermodynamical stability, the $2 \mathrm{H}$ type is dominantly formed during synthesis [57] (Fig. 8a, b). Mainly, exfoliation and filtration induced stacking of $\mathrm{MoS}_{2}$ nanosheets is similar to those of the graphene-based membranes. Recently, vapor and liquid permeation through the $\mathrm{MoS}_{2}$ lamellar structure that is comparable to graphene oxide derived membranes were reported. Lamellar (class 1) $\mathrm{MoS}_{2}$ membranes [56] showed better stability in a harsh aqueous environment (stability under a low wide range of $\mathrm{pH}$ ) than $\mathrm{GO}$ membranes while allowing permeation of light organic vapors (e.g., $\mathrm{MeOH}, \mathrm{EtOH}$, cyclohexane, and acetone) as well as water vapor. The 2D nanoscopic channel produced in the stacked $\mathrm{MoS}_{2^{--}}$ platelet membrane also suggests the molecular sieving of various ions and dye molecules. Hirunpinyopas et al. [59] reported a functionalized $\mathrm{MoS}_{2}$ lamellar membrane that effectively prevents $\mathrm{MoS}_{2}$ membranes from swelling from water exposure. Various dye molecules (crystal violet, sunset yellow, and neutral red) are used to functionalize the $\mathrm{MoS}_{2}$ surface and the effect of dye functionalization was confirmed by forward-osmosis type experiments. Notably, the $\mathrm{MoS}_{2}$ membrane functionalized by sunset yellow dye with a $5 \mu \mathrm{m}$ thickness showed a nearly $99 \% \mathrm{NaCl}$ rejection and 5 times higher water flux $\left(0.033 \mathrm{~L} \mathrm{~m}^{-2} \mathrm{~h}^{-1} \mathrm{bar}^{-1}\right)$ through the membrane than that of a similar thickness GO membrane $\left(0.007 \mathrm{~L} \mathrm{~m}^{-2} \mathrm{~h}^{-1} \mathrm{bar}^{-1}\right)$. Heiranian et al. [60] calculated the performance of class 3 type membranes formed by a single layer $\mathrm{MoS}_{2}$ membrane via $\mathrm{MD}$ simulations. Due to the sandwiched triple layer structure of the $\mathrm{MoS}_{2}$, edge site chemistry played a significant role in water permeation via the difference in hydrophobicity of different sites. By tuning the pore area of a single layer $\mathrm{MoS}_{2}$ membrane, salt rejection of the membrane reached up to $88 \%$ with water permeability comparable to that of the GO membranes. Wang et al. [61] reported the effect of hydration states on the size of the nano-channels between $\mathrm{MoS}_{2}$ layers. When the $\mathrm{MoS}_{2}$ membrane was fully hydrated, the free spacing between the layers was $0.9 \mathrm{~nm}$, while a perfectly dehydrated $\mathrm{MoS}_{2}$ membrane had a $0.3 \mathrm{~nm}$ free spacing. Fully hydrated $\mathrm{MoS}_{2}$ membranes maintained the interlayer distance by the van der Waals interaction and hydration force which created aqueous stability of the $\mathrm{MoS}_{2}$ membranes. Water molecules were transported through the fully hydrated $\mathrm{MoS}_{2}$ membranes and their membrane showed a high water flux of $30-250 \mathrm{~L} \mathrm{~m}^{-2} \mathrm{~h}^{-1}$ $\mathrm{bar}^{-1}$. However, other organic molecules (rhodamine-WT and methylene blue) are moderately cut off by their size which is larger than the free spacing between $\mathrm{MoS}_{2}$ layers. Chen et al. [62] impregnated ionic liquid (1-butyl-3-methylimidazolium tetrafluoroborate $[\mathrm{BMIM}]\left[\mathrm{BF}_{4}\right]$ ) into the interlayer of the $\mathrm{MoS}_{2}$ membranes. Due to the high solubility of $\mathrm{CO}_{2}$ toward $[\mathrm{BMIM}]\left[\mathrm{BF}_{4}\right]$, their ionic liquid confined $\mathrm{MoS}_{2}$ membranes showed noticeable selectivity for various mixtures such as $\mathrm{CO}_{2} / \mathrm{N}_{2}(\sim 131), \mathrm{CO}_{2} / \mathrm{CH}_{4}$ $(\sim 43)$ and $\mathrm{CO}_{2} / \mathrm{H}_{2}(\sim 14) . \mathrm{MoS}_{2}$ nanosheets have also been used in desalination via forward osmosis (FO) operation. Li et al. [63] stacked $\mathrm{MoS}_{2}$ nanosheets on the surface of polyethersulfone (PES) FO membrane by layer-by-layer (LBL) deposition method. The presence of $\mathrm{MoS}_{2}$ increase the hydrophilicity of the FO membrane and furthermore tuned the average pore radius that potentially affected the water permeability of the FO membrane. $\mathrm{MoS}_{2}$ coated $\mathrm{FO}$ membrane showed $35 \%$ enhanced water flux compared to that of the pristine $\mathrm{FO}$ membrane. Hydrophilic and smooth $\mathrm{MoS}_{2}$ nanochannels contribute to the higher water flux of coated FO membrane. Moreover, the reverse $\mathrm{NaCl}$ flux of $\mathrm{MoS}_{2}$ coated membrane is decreased to $16.42 \mathrm{gm}^{-2} \mathrm{~h}^{-1}, 35.91 \%$ enhanced compared to reverse $\mathrm{NaCl}$ flux of pristine PES FO membrane. Through the dynamic cross-fouling test with Bovine serum albumin (BSA) molecule, $\mathrm{MoS}_{2}$ coated FO membrane showed stronger fouling resistance than pristine PES FO membrane. $\mathrm{MoS}_{2}$ is definitely an attractive candidate for 2D-enabled membranes due to its planar characteristics of limiting defects with a uniform and well-established structure of a two dimensional transport channel.

\section{Metal organic frameworks (MOF)}

The metal organic framework (MOF) is a class of microporous material similar to the zeolite family. MOF crystals are synthesized by connecting the metal clusters by 
various multitopic organic linkers (or ligands). To date, more than 70,000 different MOFs have been experimentally confirmed in about 138,000 hypothetical frameworks [64, 65]. Unlike zeolites, which are characterized by their rigid, regular microporosity, MOFs show a higher degree of flexibility due to the rotational or translational freedom of organic linkers, such as imidazolate, carboxylate, and azolate. MOFs are frequently used as a filler for fabrication of a mixed matrix membrane due to the partial organic nature of its framework-affinity toward a polymeric matrix is higher than other purely inorganic materials. Two dimensional (MOF nanosheets) MOFs have been also reported to show favorable applications in catalysis $[66,67]$, gas storage and separations $[68,69]$, and chemical sensors [70, 71]. MOF nanosheets are prepared in two different ways: 1 ) Bottom-up synthesis (c.f., arresting crystallization) [72], 2) Top-down synthesis (c.f., exfoliation of layered MOF crystals) [73, 74] (Fig. 9). In bottom-up synthesis, a dominant growth of the 2D plane is achieved by using the appropriate ligands or surfactants. For instance, specific surfactants direct the growth of a preferential plane by their selective attachment on the specific facets of nanocrystals. Surfactant attachment on the surface of the crystal reduces the surface energy via dipole-dipole interaction, enabling the controlled growth of crystal facets [75]. Zhao et al. [76] reported the fabrication of ultrathin 2D $\mathrm{M}$-TCPP nanosheets $(\mathrm{M}=\mathrm{Zn}$ or $\mathrm{Cu}, \mathrm{TCPP}=$ Tetrakis(4-carboxyphenyl)porphyrin) using a surfactant such as poly (vinyl pyrrolidone). Conventional solvothermal synthesis without the use of a surfactant naturally creates bulk crystals of M-TCPP, but with PVP, anisotropic growth of nanocrystals is observed, and ultrathin $2 \mathrm{D}$ nanosheets with a thickness of $8.7 \pm 2.7 \mathrm{~nm}$ are formed.

a
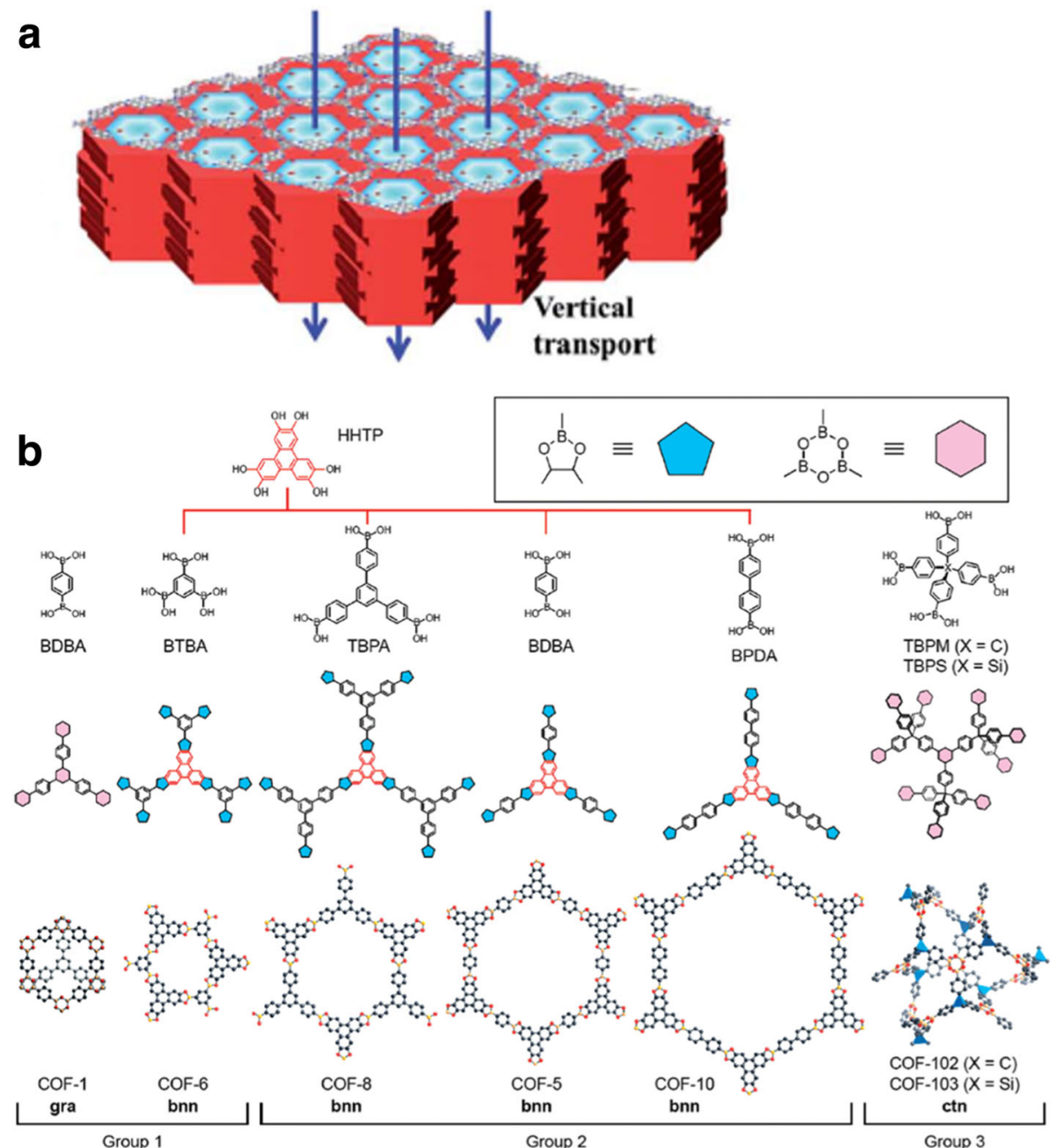

Fig. 9 a A cartoon showing the mass transport through COF membranes [86] Adapted with permission from ref [86]. Copyright 2013 for Royal Society of Chemistry. b Various example of 2D COF building blocks [164]. Adapted with permission from ref [164]. Copyright 2016 for John Wiley and Sons 
Similarly, other small modifier molecules can bind preferentially to a particular facet of growing crystals. For example, the use of pyridine in synthesizing copper based 2D MOF nanosheets [77] and the use of PVP in synthesizing cobalt based 2D MOF nanosheets have been reported [78]. 2D nanosheets of MOF can also be produced using phase-interface directed crystal growth. In the top-down synthesis of MOF nanosheets, a number of different exfoliation methods have been reported. Abherve et al. [73] mimicked the famous "scotch tape method" [79] to produce MOF nanosheets. Their MOF nanosheets had few micron lateral sizes with $2 \mathrm{~nm}$ thickness. Ball mill, grinder, stirrer, or ultrasonication have been used to mechanically exfoliate bulk layered MOFs [80-83]. Alternatively, the interlayer space in layered MOF materials can be enlarged by the intercalation of ionic/molecular species between MOF layers to enhance the delamination of the nanosheets.

These MOF nanosheets are frequently used to form class 2 or class 3 (2D) membranes. Peng et al. [80] reported $1 \mathrm{~nm}$ thick MOF nanosheets with a large lateral area and their application in fabricating molecular sieving membranes. Parent poly $\left.\left(\mathrm{Zn}_{2} \text { (benzimidazole }\right)_{4}\right) \mathrm{MOF}$ has two-dimensional layers oriented normal to the $\mathrm{c}$ axis connected by weak van der Waals interactions between layers. Wet ball milling and ultrasonication can break these weak interactions to exfoliate nanosheets and the suspension of nanosheets was further deposited onto the surface of $\alpha-\mathrm{Al}_{2} \mathrm{O}_{2}$ via a hot-drop coating method. The class 2 type membrane produced by this method had sharp molecular sieving properties with high $\mathrm{H}_{2}$ permeance (up to $3700 \mathrm{GPU}$ ) and decent $\mathrm{H}_{2} / \mathrm{CO}_{2}$ selectivity (up to 290). Similarly, Peng et al. [27] reported sub-10 $\mathrm{nm}$ thick MOF nanosheets of $\mathrm{Zn}_{2}$ (benzimidazole) ${ }_{3}(\mathrm{OH})\left(\mathrm{H}_{2} \mathrm{O}\right)$. Exfoliated MOF nanosheets were deposited using the hot-drop coating method and tested for separation of an $\mathrm{H}_{2} / \mathrm{CO}_{2}$ mixture $\left(\mathrm{H}_{2}\right.$ permeance up to 2300 GPU and $\mathrm{H}_{2} / \mathrm{CO}_{2}$ selectivity up to 166). Wang et al. [84] also used the hot-drop coating method to prepare 2D MOF membranes. 2D Mesh Adjustable Molecular Sieve (MAMS-1) nanosheets were prepared by a freeze-thaw process of the parent 3D framework. During the freeze-thaw process, the parent MAMS-1 crystals were frozen in a liquid nitrogen bath and thawed in hot water bath, repeatedly. Due to the shear force derived from the volumetric change of solvent, the 2D MAMS-1 nanosheets were exfoliated. And then a nanoscale MAMS-1 membrane was fabricated on hot anodic aluminum oxide. This membrane also selectively permeated $\mathrm{H}_{2}$ over $\mathrm{CO}_{2}$ with $\mathrm{H}_{2}$ permeance up to $800 \mathrm{GPU}$ with $\mathrm{H}_{2} / \mathrm{CO}_{2}$ selectivity up to 268 . Overall, atomically thin MOF nanosheets have been re-assembled and interlocked on porous supports to produce class 2 type membranes that combine the intrinsic micropores of MOF nanosheets and external nanopores between MOF nanosheets.

\section{Covalent organic frameworks (COF)}

Covalent organic frameworks are a novel class of microporous crystalline materials based on the integration of organic building blocks into highly ordered networks. These organic ligands are coordinated into a periodic two-dimensional networks structures to construct highly uniform micro- or mesoporous pores (Fig. 10a, b). Compared to its parent 3D structures, 2D COFs form lamellar nanosheets that can be widely exploited in class 2 or class 3 membranes. In general, Schiff base-type COFs form pore sizes in $0.8-5.0 \mathrm{~nm}$ that are useful in nanofiltration (NF) type separations, however, the pore size can further be tuned by the introduction of functional groups at the pore edge sites. Since organic linkers are covalently linked in the microporous framework, COFs show high stability under various organic conditions and further be used in organic solvent separations. A self-standing COF membrane (M-TpTD, Tp: 1,3,5-triformylphloroglucinol, TD: 4,4'-p-Phenylenedianiline) with a pore size of $2.6 \mathrm{~nm}$ showed impressive permeance for several different aprotic solvents [85]. Especially, methanol recorded the highest permeance of $138 \mathrm{Lm}^{-2} \mathrm{~h}^{-1}$ $\mathrm{bar}^{-1}$ with high rejection rate for Rose Bengal (MW: 1017.6, 99\% rejection), Congo Red (MW: 696.6, 96\% rejection) and Methylene Blue (MW: 319.8, 94\% rejection). Zhang et al. [86] used a facile bottom-up interfacial crystallization approach to obtain a 2D COF (EB-COF:Br, EB = ethidium bromide) membrane with average pore size of $1.68 \mathrm{~nm}$ and an interlayer distance of $1.65 \mathrm{~nm}$. Their membrane showed high permeance for protic solvents such as methanol $\left(1272 \mathrm{~L} \mathrm{~m}^{-2} \mathrm{~h}^{-1}\right.$ bar $^{-1}$ ), ethanol (564 $\mathrm{L} \mathrm{m}^{-2} \mathrm{~h}^{-1} \mathrm{bar}^{-1}$ ) and $n$-propanol (477 $\mathrm{L} \mathrm{m}^{-2} \mathrm{~h}^{-1} \mathrm{bar}^{-1}$ ), and for aprotic solvents such as acetone $\left(2640 \mathrm{~L} \mathrm{~m}^{-2} \mathrm{~h}^{-1} \mathrm{bar}^{-1}\right)$, acetonitrile $\left(2095 \mathrm{~L} \mathrm{~m}^{-}\right.$ $\left.{ }^{2} \mathrm{~h}^{-1} \mathrm{bar}^{-1}\right)$. Interestingly, near perfect $(\sim 100 \%)$ rejection for anionic dyes (Methyl Orange, Fluorescein Sodium salt, Potassium Permanganate) due to the strong electrostatic interaction with the positively charged pore walls of COF. Shinde et al. [87] used the Langmuir-Blodgett (LB) method to fabricate 2D COF membrane with crystalline TFP-DHF and performed organic solvent nanofiltration. $\beta$-ketoenamine linkages between TFP and DHF are formed and inclined AA stacked hexagonal lattice COF with the pore size of 1.41 $\mathrm{nm}$ are synthesized. Through the LB method, membrane thickness can be controlled precisely. Their membrane showed large high contact angle $\left(84.5^{\circ}\right)$ and thermal stability up to $200^{\circ} \mathrm{C}$. Previously, imine bonded two-dimensional COF membranes (thickness of 1.7, 2.3, and $4.7 \mathrm{~nm}$ ) by using LB method were reported [88]. Compared to the previously reported membrane, their 


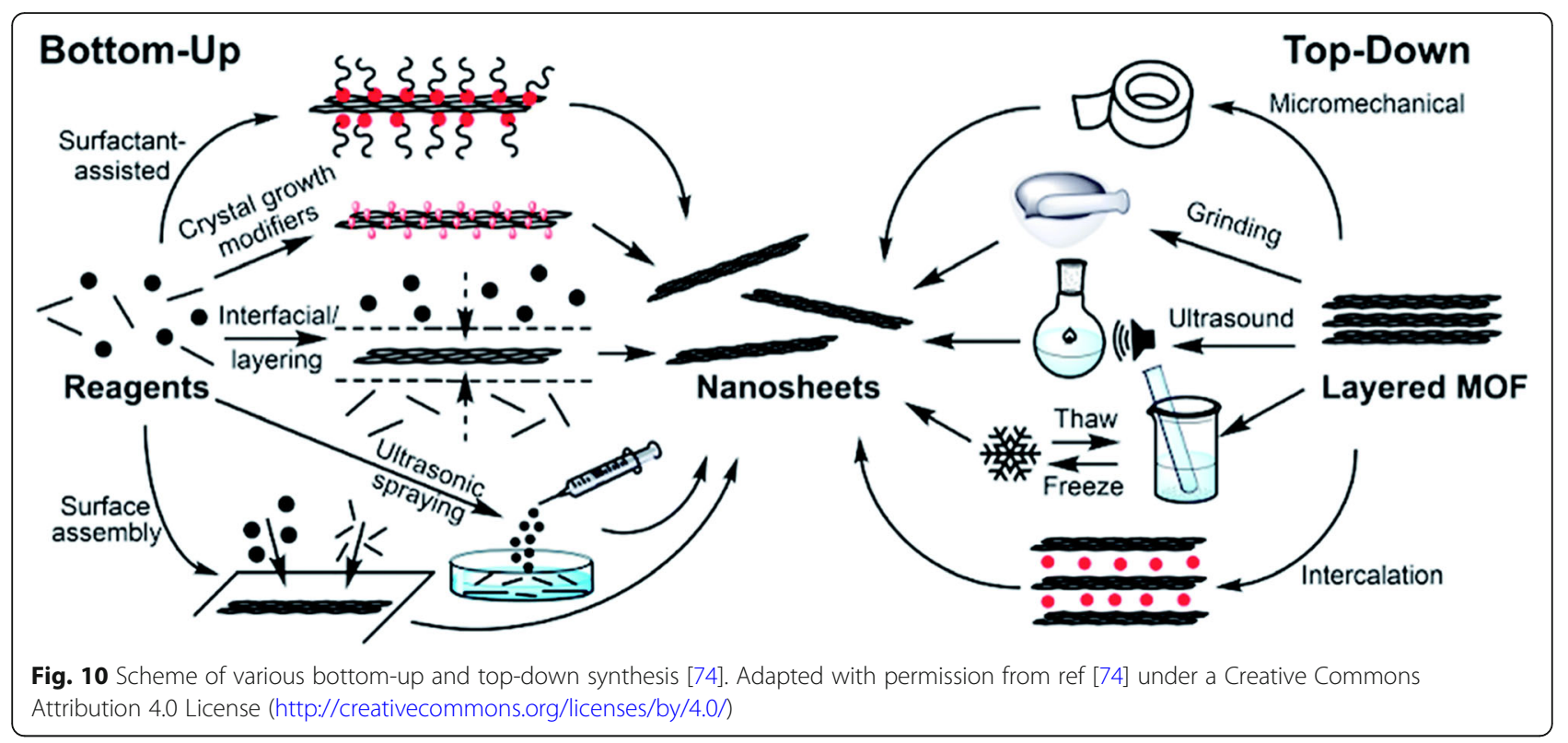

newly fabricated membrane showed 100 times higher solvent permeability. The molecular weight cutoff (MWCO) of TFP-DHF 2D COF membrane showed around $900 \mathrm{Da}$ and the molecular weight retention onset (MWRO) of the membrane is around $600 \mathrm{Da}$. Zhang et al. [89] reported MD simulation of 2D COF membrane for desalination. The triformylphloroglucinol (Tp) and p-phenylenediamine $(\mathrm{Pa})$ with several functional groups (X) are used to fabricate 2D COF membrane (TpPa-X) by tuning the aperture sizes in the range of 5.1-7.6. Hydrophilic functional groups with larger aperture size were advantageous in high water permeance of the $2 \mathrm{D}$ COF membranes. Through MD simulation, their 2D COF based membranes showed 1-2 orders of magnitude higher water permeance compared to that of 3D ZIF membranes, which have totally opposite structure to $2 \mathrm{D}$ COF membrane, with relatively long channels.

In addition to organic solvent separations, various gas separation membranes are also demonstrated using 2D COF nanosheets. Li et al. [90] exfoliated microporous COF-1 nanosheets (average pore size $\sim 0.39 \mathrm{~nm}$ ) to fabricate ultrathin COF-1 membrane (COF nanosheets layer of $\sim 100 \mathrm{~nm}$ ). The COF-1 membrane was fabricated through solvothermal condensation of 1,4-benzenediboronic acid (BDBA) in a 1,4-dioxane-mesitylene mixture. Their membrane showed the $\mathrm{H}_{2}$ permeance of $10^{-6} \mathrm{~mol} \mathrm{~m}^{-2} \mathrm{~Pa}^{-1} \mathrm{~s}^{-1}$ (3000 GPU), which surpass the performance of ZIF- 8 membrane with $100 \mathrm{~nm}$ thickness (5.46 $\left.\times 10^{-8} \mathrm{~mol} \mathrm{~m}^{-2} \mathrm{~Pa}^{-1} \mathrm{~s}^{-1}, 163 \mathrm{GPU}\right)$. Tong et al. [91] reported molecular dynamics simulation of triazine-based 2D COF membranes (CTF-1) with an average pore size of $12 \AA$. With different stacking mode, gate closing effect was studied by varying the interlayer distance of COF nanosheets. They simulated the transport behavior of $\mathrm{CO}_{2} / \mathrm{N}_{2}$ mixture through their membrane. By tuning the structure of four-layered $2 \mathrm{D}$ COF membrane, high $\mathrm{CO}_{2} / \mathrm{N}_{2}$ selectivity up to 36 was obtained while maintaining the $\mathrm{CO}_{2}$ flux of 23 molecules/ns. Through their work, tuning the stacking mode of nanosheets can be one of the most effective strategies to fabricate the ultrathin membrane with high permeance and selectivity. Due to its inherently large pore sizes (Schiff based COFs), 2D COF based membranes show preferential applications in organic solvent separations. Pharmaceutical industries or biorefinery where there is a need for solvent-resistant membranes with high permeance of organic solvents are expected to be positively impacted by $2 \mathrm{D} \mathrm{COF}$ membranes.

\section{Basal plane engineering and surface chemistry of 2D nanocomposite materials}

Surface functionalization of GO

The surface characteristics of graphene oxide $(\mathrm{GO})$ has been extensively investigated owing to its unique properties: a tunable chemical structure, pore generation, high permeability and single-atomic thickness with lateral dimensions [92]. It is well-known that GO presents various oxygen-containing functional groups, such as hydroxyl, epoxy, carbonyl, and carboxylic acid groups, on its surfaces, which can be reacted with their corresponding chemical reactions to fabricate high-performance membranes. Those functional groups can be chemically modified with other functional groups using various chemical reactions: amide coupling [93], nucleophilic substitution [94], diazotization [95], silylation [96], etc. (Fig. 11a). Since there are several focused reviews on the surface chemistry of GO sheets, we focus on the surface modifications of $\mathrm{GO}$ for membrane 
applications. The amide coupling reaction has been frequently used to functionalize GO sheets with primary amine groups to introduce positive charges on their surface in a colloidal state, and the negatively charged GO and positively charged $\mathrm{GO}-\mathrm{NH}_{2}$ was utilized as a building block to construct multi-layered structures of $\mathrm{GO}$ sheets through a layer-by-layer (LbL) assembly process to fabricate a membrane for durable reverse-osmosis application [97]. The resulting LbL multi-layers on a polyamide membrane resulted in significantly improved antifouling properties against proteins and the resistance of membrane degradation by chlorine during a salt rejection process. This amide coupling strategy can be also used to cross-link the assembled GO sheets with lamella-like structures by inter-connection of carboxylic acid groups of GO sheets to improve their mechanical stability. For example, the laminated $\mathrm{GO}$ sheets on a polycarbonate membrane were spontaneously delaminated in aqueous media by brief sonication or incubation in basic conditions. However, the cross-linked GO laminates with branched polyethyleneimine (BPEI) were stable in a wide range of $\mathrm{pH}$ values with prolonged sonication up to $1 \mathrm{~h}$, and the BPEI cross-linking process did not affect the filtration performance of laminated GO sheets, such as the rejection rate against both negatively and positively charged organic dye molecules [98]. The nucleophile substitution reaction was harnessed for carboxylation of $\mathrm{GO}$ and the carboxylated $\mathrm{GO}$ $(\mathrm{GO}-\mathrm{COOH})$ presented higher water dispersibility and chemical tailorability than pristine GO owing to the increased density of carboxylic acid groups. When the $\mathrm{GO}-\mathrm{COOH}$ prepared by reaction with glycine was




laminated on a polyethersulfone (PES) membrane, its laminated structures provided many wrinkles and hydrophilic surface properties, which was different from pristine GO, which acts as an active water transport channel [99]. As a result, the laminated $\mathrm{GO}-\mathrm{COOH}$ provided a prominent water flux with improved desalting properties by enhancement of electrostatic repulsion (Fig. 11b). The silylation is also an important chemical reaction in modifying GO with desirable functional groups [96]. As GO presents many hydroxyl groups on its basal plane, it can be easily reacted with diverse silane compounds presenting different functional groups [100]. For the membrane application, the surface of GO was modified with $\mathrm{N}$-(trimethoxysilylpropyl) ethylenediamine triacetic acid (EDTA-silane) to increase the density of carboxylic acid groups [101]. The EDTA-silane functionalized GO (EDTA-GO) exhibited much higher aqueous dispersibility than pristine GO, and furthermore, it can be stably dispersed in water even after chemical reduction (EDTA-RGO). The laminated EDTA-RGO showed high applicability for ion separation with a significantly reduced permeation rate of small cations such as $\mathrm{K}^{+}$and $\mathrm{Na}^{+}$(Fig. 11c). The non-covalent approach is another effective strategy for surface functionalization of GO through various secondary interactions, such as electrostatic, hydrogen bonding and hydrophobic interaction (Fig. 11a). GO has a highly negative-charged surface and can be utilized to construct laminated structures by an LbL assembly process with poly (allylamine hydrochloride) (PAAH) [102] and gelatin [103]. The resulting laminated structures exhibited considerably improved performance for water purification and mixed solvent separation. The surface functionalization of GO with a cationic surfactant through electrostatic and hydrophobic interactions improved the forward osmosis process [104].

\section{Surface functionalization of $\mathrm{MoS}_{2}$}

$\mathrm{MoS}_{2}$ is one of the most representative TMDs owing to its physicochemical properties such as single-atomic thickness, tailorable surface, thermal and chemical stability [105]. Despite the high potential, the membrane application of $\mathrm{MoS}_{2}$ has been relatively restricted owing to the difficulty for large-scale preparation of single-layered sheets with a large lateral dimension, but this problem is being addressed by many efforts to improve the exfoliation and functionalization processes of bulk $\mathrm{MoS}_{2}$. Although there is still a debate on the functionalization mechanism, it is well-known that the surface of $\mathrm{MoS}_{2}$ can be readily functionalized with thiol compounds by physisorption or direct coordination to $\mathrm{S}$ vacancies of $\mathrm{MoS}_{2}$ (Fig. 12a) [106, 107]. The functionalized $\mathrm{MoS}_{2}$ sheets presented diverse surface properties depending on the terminal groups of thiol ligands, and thus they were highly dispersible in a complex aqueous media [108]. In addition, since the surface of $\mathrm{MoS}_{2}$ sheets was partially oxidized during the exfoliation process, their surface was easily functionalized by a sol-gel reaction with silane compounds [109] or electrostatic interaction of polymers [63] and small molecules [110]. The non-covalent functionalization of $\mathrm{MoS}_{2}$ with poly organic dyes also greatly improved the membrane performance of laminated $\mathrm{MoS}_{2}$ sheets over their pristine counterpart [59]. These previous reports indicated the importance of surface functionalization of $\mathrm{MoS}_{2}$ sheets for application as a membrane material.

\section{Surface functionalization of $\mathrm{h}-\mathrm{BN}$}

h-BN is an attractive 2D material for membrane applications owing to its unique and excellent properties such as high strength, insulation, thermal stability and conductivity and chemical inertness [111-113]. Those properties make it promising, but its extremely high chemical inertness has restricted its wide-spread application. Surface functionalization strategies for h-BN can be also classified into covalent and non-covalent approaches. The covalent approach is generally a one-step process in which the exfoliation and functionalization of BN sheets occur simultaneously. The aqueous exfoliation and functionalization of h-BN sheets with hydroxyl groups was achieved through a hydrolysis reaction by prolonged-sonication of $\mathrm{h}$ - $\mathrm{BN}$ in aqueous media (Fig. 12b) [114], refluxing in concentrated strong acids or bases $[115,116]$, solution-phase oxygen radical reaction [117], using urea with a ball milling process, using molten hydroxides [118], or direct hydrothermal reaction with hydrogen peroxide. The hydroxylated h-BN sheets can be diversely derivatized using a silylation reaction with various silane compounds [119, 120]. Despite those efforts, the membrane applications of h-BN sheets were restricted owing to their low exfoliation and production yield. By contrast, Lei et al. reported an efficient strategy to synthesize amine-functionalized h-BN $\left(\mathrm{BN}-\mathrm{NH}_{2}\right)$ on a large scale by ball-milling h-BN powder with urea, and the resulting $\mathrm{BN}-\mathrm{NH}_{2}$ sheets were stably dispersed at a high concentration in aqueous media (Fig. 12c) [121]. Recently, they also demonstrated that the laminated $\mathrm{BN}-\mathrm{NH}_{2}$ sheets can be harnessed as a stable and efficient molecular separation membrane for molecules dissolved in both aqueous and organic solvents with a high permeation rate under acidic, alkaline and oxidative conditions [50,54]. In addition to laminated $\mathrm{BN}-\mathrm{NH}_{2}$ sheets, it was also revealed that $\mathrm{BN}-\mathrm{NH}_{2}$ sheets can be directly composited with polypiperazine (PPA) by interfacial polymerization, and the resulting composite membrane exhibited a $13.5 \%$ higher permeation rate than a bare PPA membrane without 

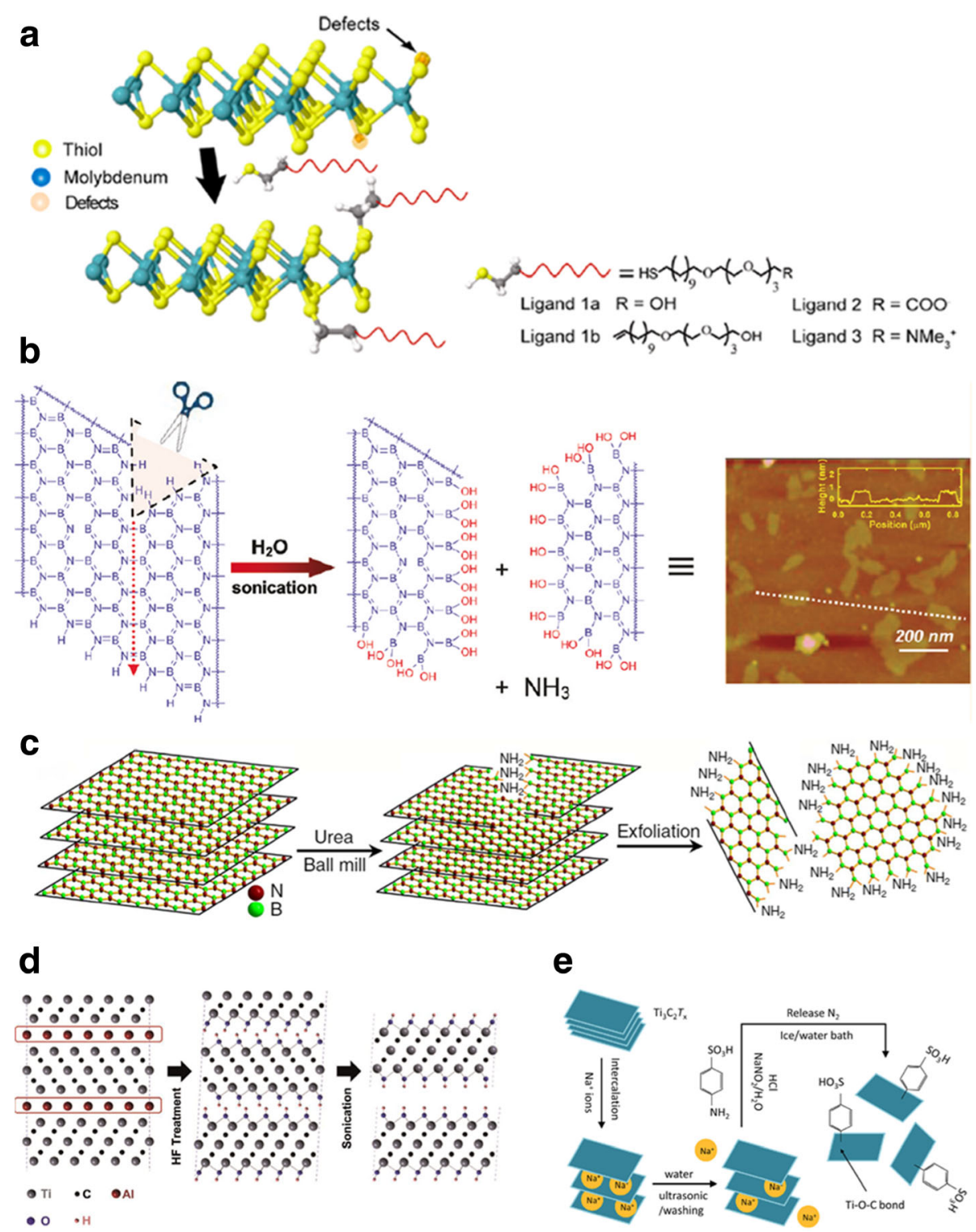

Fig. 12 a Surface functionalization of $\mathrm{MoS}_{2}$ sheets with thiol compounds [107]. Adapted with permission from ref [107]. Copyright 2013 for American Chemical Society. $\mathbf{b}$ Exfoliation and hydroxyl functionalization process of h-BN sheets by sonication-assisted hydrolysis [114]. Adapted with permission from ref [114] Copyright 2011 for American Chemical Society. c Exfoliation and amine functionalization of h-BN sheets by ureaassisted ball milling [121]. Adapted with permission from ref [121]. Copyright 2015 for Nature Publishing Group. $\mathbf{d}$ Exfoliation process of $\mathrm{Ti}_{3} \mathrm{C}_{2} \mathrm{~T}_{\mathrm{x}}$ sheets by chemical etching of MAX phases by using a fluoride-containing agents [126]. Adapted with permission from ref [126]. Copyright 2011 for John Wiley and Sons. e Functionalization of $\mathrm{Ti}_{3} \mathrm{C}_{2} \mathrm{~T}_{x}$ sheets with phenylsulfonic groups by diazotization [130]. Adapted with permission from ref [130]. Copyright 2018 for Elsevier

compromising rejection properties against organic molecules [122].

\section{Surface functionalization of $M X e n e\left(\mathrm{Ti}_{3} \mathrm{C}_{2} \mathrm{~T}_{\mathrm{x}}\right)$}

$\mathrm{Ti}_{3} \mathrm{C}_{2} \mathrm{~T}_{\mathrm{x}}$ is a representative material of the MXene family and the laminated MXene $\left(\mathrm{Ti}_{3} \mathrm{C}_{2} \mathrm{~T}_{\mathrm{x}}\right)$ sheets have been spotlighted as a promising material for membrane applications based on their well-ordered subnanometer channels [42], hydrophilicity, high water flux [123], salt rejection [124], and anti-biofouling properties [125]. MXene sheets have been generally synthesized by chemical etching of MAX phases using fluoride-containing agents (Fig. 12d), including $\mathrm{HF}[126,127], \mathrm{LiF} / \mathrm{HCl}$ [128], $\mathrm{NaHF}_{2}, \mathrm{Na}_{4} \mathrm{HF}_{2}$, and $\mathrm{KHF}_{2}$ [129]. During the etching process, the composition of terminal $-\mathrm{O},-\mathrm{OH}$ and $-\mathrm{F}$ groups on MXenes varied with the chemical etchants, and it was recently found that $\mathrm{LiF} / \mathrm{HCl}$ is an efficient etchant to synthesize MXene sheets with a large 
lateral dimension and a single-layered and $-\mathrm{OH}$ group terminated structure [128]. Therefore, the etching process can be regarded as a simultaneous process of exfoliation and surface functionalization for the large-scale production of hydroxylated MXene sheets. The surface of MXene sheets can be further modified with covalent and non-covalent approaches like graphene derivatives. As a covalent approach, Wang et al. reported that the diazotization reaction of MXene sheets sulfanilic acid diazonium salts resulted in enhanced colloidal stability [129] and electrochemical activity (Fig. 12e) [130]. In the case of non-covalent approaches, MXene sheets present a negative surface charge owing to their hydroxyl groups, and thus they can have electrostatic and hydrogen-bonding interactions with poly (diallyldimethylammonium chloride) (PDDA) and polyvinyl alcohol (PVA) [131]. In the same line, the surface of MXenes can be functionalized with polyethyleneimine (PEI) and polydimethylsiloxane (PDMS) and the resulting laminates of PEI- and PDMS-functionalized MXene sheets achieved improvement in mechanical/thermal stability and solvent resistances accompanied by the enhanced transfer of alcohols and the rejection rate of solutes [132]. In addition, the complexation of positively charged Fe $(\mathrm{OH})_{3}$ colloids with MXene sheets through electrostatic interaction resulted in the formation of expanded nanochannels and then enhancement of the water flux and rejection rate for approximately 2.5 nm-sized molecules [133]. Those previous studies clearly indicated that the surface functionalization of MXene sheets provide more opportunities to develop a multifunctional and high-performance membrane material.

\section{Nanocomposite membranes formed with 2D materials}

Nanocomposites formed with 2D materials are also an extended version of the $2 \mathrm{D}$-enabled membranes. At a high-level, these nanocomposites can be classified into two types: 1) Polymer-based nanocomposites and 2) 2D nanocomposites formed with elements other than polymers $(\mathrm{GO}+\mathrm{MOF}, \mathrm{COF}+\mathrm{MOF}$, or $\mathrm{COF}+\mathrm{COF}$...). In polymer-based nanocomposites formed with $2 \mathrm{D}$ materials, the mixed-matrix membrane (MMM) architecture and the thin film composite (TFC) are the most well-known and studied membrane structure. Thin film composite (TFC) is one of the most representative asymmetric membrane architecture that is made of very thin (usually less than $200 \mathrm{~nm}$ ) dense polymeric layer (separating layer) is deposited on top of a porous support. Polyamide composited with polyethersulfone (PES) or polysulfone (PS) support membrane is widely used in water purification or desalination systems. For mass production of TFC membrane, various scalable fabrication techniques have been reported such as dual-layer slot coating [134] or the hollow fiber spinning [135]. Compared to TFC membranes, MMMs have better thermal and mechanical stability arising from incorporated nanoparticles (fillers) that reduce the impact of heating and membrane compaction. On the other hands, TFC membranes are described by much thinner separating layer thickness compared to MMMs, TFC membranes are often referred to as high permeance membranes. However, TFC membranes typically suffer from membrane compaction and fouling. To overcome these limitations, TFC membranes are modified with fillers (similar to MMM). The following TFC based composite membranes using 2D fillers have been reported. Li et al. [136] fabricated TFC reverse osmosis membrane modified by 2D laminar $\mathrm{MoS}_{2}$. Due to membrane surface modification of $\mathrm{MoS}_{2}$ nanosheets, the surface of $\mathrm{MoS}_{2}$-TFC membrane showed improved hydrophilicity and roughness. As a result, $\mathrm{MoS}_{2}$-TFC membrane showed high water flux with the anti-fouling property. Dong et al. [137] modified TFC by clay nanosheets. A cationic clay (montmorillonite, MMT) and an anionic clay (layered double hydroxide, LDH) were used for modification of TFC. Likewise, clay-TFC showed hydrophilicity and anti-fouling property because of hydrophilic clays. Especially, MMT and LDH incorporated TFC membranes showed significantly improved the anti-fouling property to protein and cationic surfactant due to their negatively charged surface.

The original concept of the mixed-matrix membrane has evolved from the idea that incorporated filler materials can enhance either the permeability or the selectivity of the polymeric matrix-eventually overcoming the selectivity-permeability trade-off of conventional polymer based membranes. A wide range of fillers has been adopted in MMMs, ranging from nonporous inorganic nanoparticles to highly microporous frameworks including, MOFs and zeolites. Two dimensional materials (graphene, graphene oxide, MXene, 2D MOF, 2D zeolite ...) with a high aspect ratio can also be incorporated into a mixed-matrix membrane opening up the possibility to form "ultrathin" composite membrane layers. These 2D fillers provide a tortuous diffusion pathway via stacked lamellar structures. Moreover, it is possible for few nano-meter thick 2D fillers to form a mixed-matrix with a larger volume fraction, which is not possible with the conventional 3D shape fillers.

\section{GO based MMMs}

Among various 2D materials, the graphene and graphene oxide family are the most studied materials in nanocomposites such as mixed-matrix membranes. In particular, due to its affinity toward a polymeric matrix and versatile functionality, graphene oxides have been one of the most popular filler choices in the fabrication 
of MMM. One important issue related to $\mathrm{GO}$ synthesis is the non-uniform lateral sizes of the fabricated GO nanosheets. Shen et al. [138] showed the size effects of graphene oxide on the separation performance of MMMs using the small (100-200 nm)-/medium (1$2 \mu \mathrm{m})$-/large $(5-10 \mu \mathrm{m})$-lateral sized GO. Researchers used polyether-block-amide (PEBA, Pebax ${ }^{\circ}$ ) as a polymeric matrix and investigated the chain mobility of the GO incorporated polymer matrix. The mobility of the polymer chains was restricted with increasing lateral size of GO, while large-sized GO showed poor dispersion properties in a polymer solution. MMM fabricated with 1-2 $\mu \mathrm{m}$ lateral sized $\mathrm{GO}$ exhibited excellent and stable separation of the $\mathrm{CO}_{2} / \mathrm{N}_{2}$ pair with $\mathrm{CO}_{2}$ permeability up to 110 Barrer and $\mathrm{CO}_{2} / \mathrm{N}_{2}$ selectivity up to 80 . Dong et al. [139] reported the use of porous reduced graphene oxide obtained by a dehydration reaction using $\mathrm{NaOH}$ as a filler for MMM fabricated with PEBA (Pebax $\left.{ }^{\odot}\right)$. Incorporation of the lamellar structure of the porous rGO was advantageous in selectivity enhancement for the
$\mathrm{CO}_{2} / \mathrm{N}_{2}$ pair, since the hydrated lamellar structure of rGO favorably adsorbed $\mathrm{CO}_{2}$ over $\mathrm{N}_{2}$. Furthermore, the reduction degree of the porous $\mathrm{rGO}$ controlled the permeability through the composite membrane by creating enlarged transport pathways for gas molecules. In summary, their MMM showed $\mathrm{CO}_{2}$ permeability up to 119 Barrer and $\mathrm{CO}_{2} / \mathrm{N}_{2}$ selectivity up to 100 . Shen et al. [140] reported polyvinylamine (PVAm) and chitosan (Cs) based MMM using graphene oxide grafted with hyperbranched polyethyleneimine (HPEI-GO). Amine functionalization via incorporation of HPEI on GO sheets not only enhanced the polymer-GO adhesion properties but also increased the $\mathrm{CO}_{2}$ sorption capacity through reversible chemisorption. Their membrane showed a slightly increased $\mathrm{CO}_{2} / \mathrm{N}_{2}$ selectivity $(\sim 107)$ with moderate-to-low permeance of $36 \mathrm{GPU}$. Li et al. [141] introduced the polyethylene glycol- and polyethyleneimine-functionalized GO sheets (PEG-PEI-GO) to form a mixed-matrix membrane with Pebax ${ }^{\circ}$. Ethylene oxide and amine functional groups

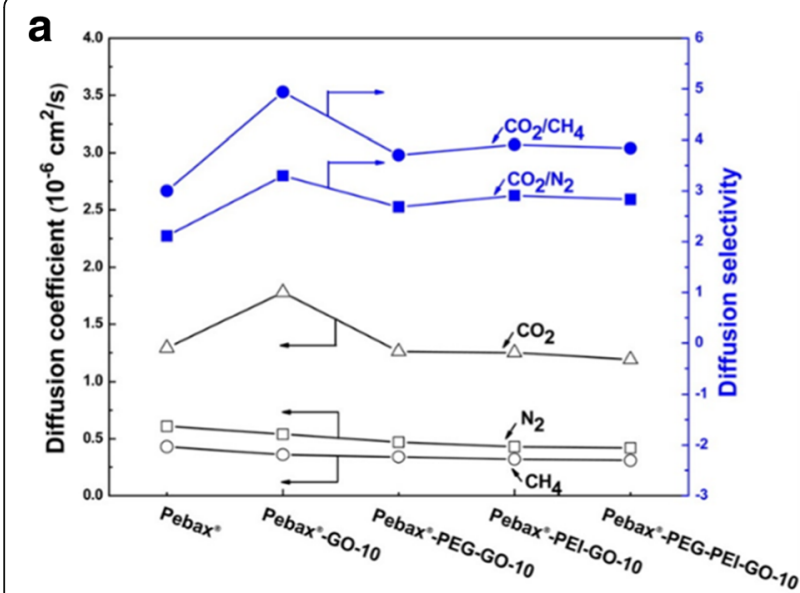

b

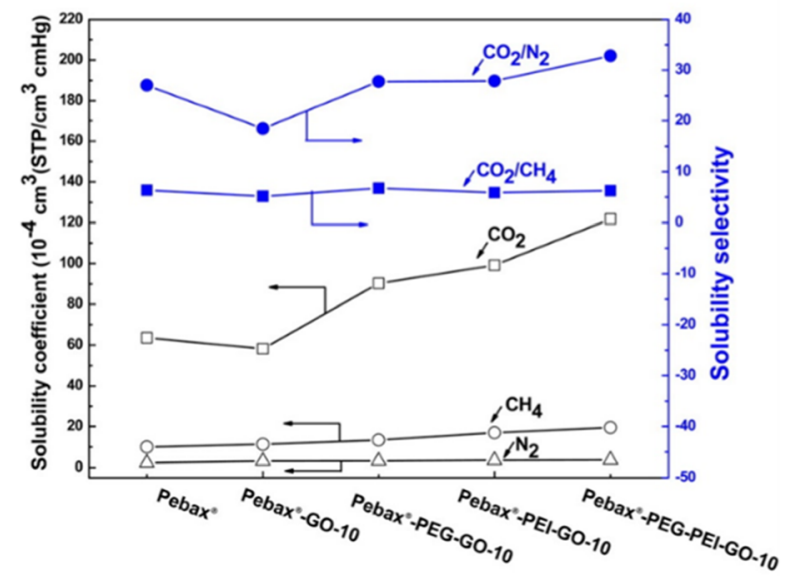

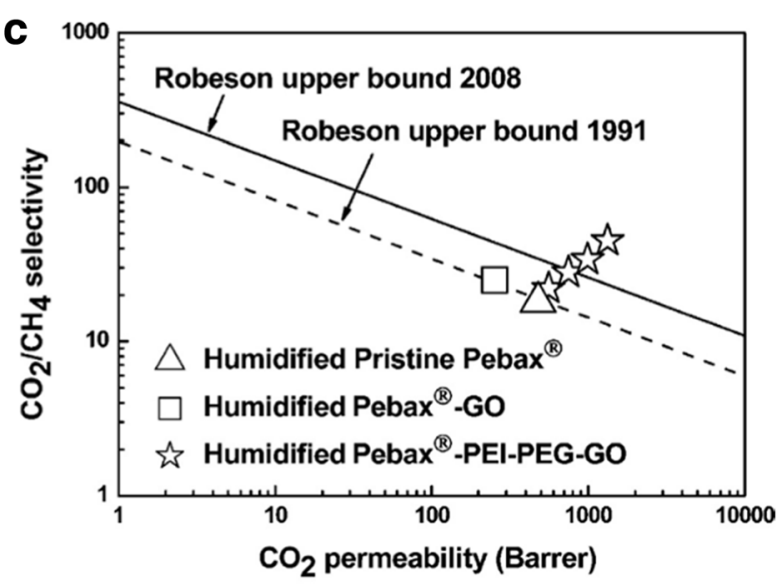

d

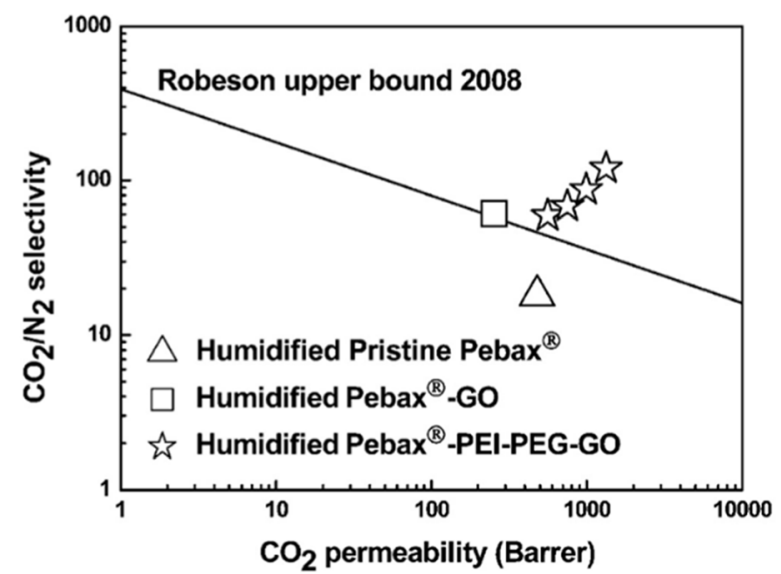

Fig. 13 a Diffusion coefficient and selectivity, $\mathbf{b}$ solubility coefficient and selectivity of $\mathrm{N}_{2}$, $\mathrm{CH}_{4}$, and $\mathrm{CO}_{2}$ in pure polymer membrane, $\mathrm{GO}$ based MMM and functionalized $\mathrm{GO}$ based MMM. c Robeson plot for $\mathrm{CO}_{2} / \mathrm{CH}_{4}$ separation. d Robeson plot for $\mathrm{CO}_{2} / \mathrm{N}_{2}$ separation [141]. Adapted with permission from ref [141]. Copyright 2015 for American Chemical Society 
from PEG and PEI, respectively, had an excellent affinity toward $\mathrm{CO}_{2}$. The mixed-matrix membrane showed a much-improved separation performance with $\mathrm{CO}_{2}$ permeability up to 1330 Barrer with $\mathrm{CO}_{2} / \mathrm{CH}_{4}$ selectivity up to 45 and $\mathrm{CO}_{2} / \mathrm{N}_{2}$ selectivity up to 120 (Fig. 13).

Due to the hydrophilicity of the GO sheets, mixed-matrix membranes formed with GO generally have good aqueous or liquid stability, and GO based MMMs are frequently used in water purification or pervaporative separations. The current state-of-the-art membrane process for desalination is illustrated by the interfacial-polymerized polyamide thin film composites. Yin et al. [142] constructed a polyamide thin-film nanocomposite using a GO based polyamide mixed-matrix membrane. Typical interfacial polymerization of polyamide film was performed on the polysulfone support layer with m-phenylenediamine (MPD) and trimesol chloride (TMC). GO nanosheets were dispersed in a TMC-hexane solution layer and the GO was incorporated into the polymeric matrix of the polyamide thin film. Incorporation of the GO nanosheets enhanced salt rejection ( $\mathrm{NaCl}$ : 93.8\%, $\mathrm{NaSO}_{4}: 97.3 \%$ ) and water flux (59.4 $\left.\mathrm{Lm}^{-2} \mathrm{~h}^{-1}\right)$ through restricted interlayer spacing ( $0.83 \mathrm{~nm}$ ) of the lamellar structure and further increased the water permeability through the hydrophilic water channel of the GO. Instead of forming a thin-film composite, GO nanosheets were also exploited in a conventional MMM platform for water purification. Ganesh et al. [143] reported the salt rejection of GO based MMM using polysulfone as a polymeric matrix. Insertion of GO into the polymeric matrix have induced the formation of macrovoids in membranes, which is responsible for high water flux. In addition, Yang et al. [144] used graphene dispersed polydimethylsiloxane (PDMS) MMM for pervaporative desulfurization. Due to the abundant population of the $\pi$ electrons on the graphene nanosheets, $\mathrm{CH}-\pi$ interaction between the high aspect ratio graphene and the PDMS chain was available. A $\pi-\pi$ interaction between the thiophene and graphene was also constructed, and a favorable interfacial structure between GO and PDMS was consequently formed. The GO/PDMS membrane showed an improved flux of $\sim 6$ $\mathrm{kg} \mathrm{m}^{-2} \mathrm{~h}^{-1}$ and a selectivity of $\sim 3.5$ for applications in pervaporative desulfurization as well as separation of aromatics-containing mixtures.

\section{D MOF based MMMs}

Microporous materials have also been a popular choice for filler material in the fabrication of mixed-matrix membranes. Among various microporous frameworks, MOFs have distinctive advantages over classical zeolite families due to their favorable interaction with a polymeric matrix. MOFs form highly dispersed polymer solutions and can be fabricated into a dense film with significantly reduced interfacial gaps [145]. On the other hand, conventional zeolites form a "sieve-in-a-cage" structure due to its immiscibility with the organic phase [146] (c.f., a polymeric matrix). Rodenas et al. [147] reported the $\mathrm{Cu}$ (BDC) (BDC: 1,4-benzenedicarboxylate) MOF based mixed-matrix membrane for $\mathrm{CO}_{2} / \mathrm{CH}_{4}$ separation especially focusing on the use of MOF nanosheets as a filler. Bulk (3D) MOF crystals leave a significant fraction of the composite volume unoccupied due to their bulky nature, but 2D MOF lamellae are uniformly distributed over the entire MMM. This uniform distribution of nanosheets throughout the matrix is translated as improved separation performance by eliminating the unselective transport pathways. Moreover, unlike isotropic fillers (nanocrystals or bulk MOF crystals) that cause swelling of the polymer matrix on the uptake of the highly plasticizing $\mathrm{CO}_{2}$, nanosheet loaded MMMs effectively suppressed the swelling and therefore maintained a high $\mathrm{CO}_{2} / \mathrm{CH}_{4}$ separation factor (up to 90) at a transmembrane pressure up to 8 bar. Shete et al. [148] also reported the use of $\mathrm{Cu}(\mathrm{BDC})$ nanosheets in mixed-matrix membranes. $\mathrm{Cu}(\mathrm{BDC})$ nanosheets were prepared in the bottom-up direct synthesis and the synthesized nanosheets had aspect ratios as high as 100 (average lateral size $2.5 \mu \mathrm{m}$ and thickness of $25 \mathrm{~nm}$ ). Polyimide $\left(\right.$ Matrimid $^{\circ}$ ) was used to provide a polymeric matrix and a $\mathrm{Cu}$ (BDC) nanosheet dispersed MMM showed much-improved separation performance in terms of $\mathrm{CO}_{2} / \mathrm{CH}_{4}$ selectivity compared to neat polyimide membranes. Jungle-gym-like [149] $\mathrm{Cu}_{2}(\mathrm{ndc})_{2}(-$ dabco) (ndc: 1,4-naphthalenedicarboxylate, dabco: 1.4-diazabicyclo[2.2.2] octane) nanosheets were reported and these nanosheets were blended with polybenzimidazole (PBI) to form a mixed-matrix membrane used in a pre-combustion $\mathrm{CO}_{2}$ capture process. Similar to $\mathrm{Cu}$ (BDC), $\mathrm{Cu}_{2}(\mathrm{ndc})_{2}$ (dabco) nanosheets showed a larger improvement when incorporated into a MMM compared to bulk 3D crystals. For a high-pressure (up to 5 bars of $\mathrm{CO}_{2}$ ) pre-combustion $\mathrm{CO}_{2}$ capture process, the mixed-matrix membrane showed $\mathrm{H}_{2}$ permeability up to 6.1 Barrer with $\mathrm{H}_{2} / \mathrm{CO}_{2}$ selectivity up to 26 . The concept of the mixed-matrix membrane has been further expanded to microporous, rigid polymers such as "polymers of intrinsic microporosity (PIMs)". Cheng et al. [150] reported a PIM-1 based mixed-matrix membrane with $\mathrm{Cu}$ (BDC) nanosheets. Notably, ultrathin mixed-matrix membranes with a thickness of $660 \mathrm{~nm}$ have been fabricated with $2 \mathrm{D}$ nanosheets and achieved high $\mathrm{CO}_{2}$ permeance (up to $400 \mathrm{GPU}$ ) with moderate $\mathrm{CO}_{2} / \mathrm{CH}_{4}$ selectivity (up to 15 ). MMMs with a thickness of $60 \mu \mathrm{m}$ had a relatively low performance in terms of $\mathrm{CO}_{2}$ permeance (up to $196 \mathrm{GPU}$ ) with low $\mathrm{CO}_{2} / \mathrm{CH}_{4}$ selectivity. The effect of nanosheets on separation performance of MMMs is dramatically compared further by 
two model MOFs. Samarasinghe et al. [151] fabricated MMMs containing both 2D nanosheets $(\mathrm{Cu}(\mathrm{BDC}))$ and 3D bulk crystals (ZIF-8, ZIF: zeolitic-imidazole framework). MMMs were successfully fabricated without significant defects and showed both enhanced $\mathrm{CO}_{2}$ permeability and $\mathrm{CO}_{2} / \mathrm{CH}_{4}$ permselectivity compared to a filler-free polymeric membrane. By incorporating the $2 \mathrm{D}$ and $3 \mathrm{D}$ fillers together, the disadvantage of the $2 \mathrm{D}$ filler (low $\mathrm{CO}_{2}$ diffusivity) can be easily overcome as $3 \mathrm{D}$ filler can increase $\mathrm{CO}_{2}$ diffusivity. Moreover, the MMM containing both $2 \mathrm{D}$ and $3 \mathrm{D}$ fillers incorporated the benefit of the $2 \mathrm{D}$ fillers, which is the effective enhancement of selectivity. As a result, this MMM showed improved separation performance with high permeability (3D fillers) and high selectivity (2D fillers).

\section{MXene based MMMs}

Similar to other 2D materials, single- or few-layered flakes of MXene nanosheets are capable of forming nanocomposites with polymers. The hydrophilic nature of the MXene nanosheet makes it possible to form a highly stable dispersion in various aqueous or organic media (e.g., aprotic polar solvents) to fabricate 2D nanocomposite membranes [152]. Formation of the highly stable dispersion in organic solvents is directly translated into the formation of non-segregating polymer solutions for further scalable membrane processing. Moreover, as discussed in the earlier sections, the lamellar structure of the 2D MXenes are characterized by the extremely short transport pathway and the large volume of the nanochannel, and nanocomposite membranes based on MXene nanosheets are able to enhance membrane separation performances. Therefore, MMMs based on MXene nanosheets often show very high water permeance (> $1000 \mathrm{Lm}^{-2} \mathrm{~h}^{-1} \mathrm{bar}^{-1}$ ) with a favorable dye rejection rate $(90 \%$ for Evans blue of molecular weight 960 , 97\% for Cytochrome C of molecular weight 12,000) [133]. Due to its stability under harsh chemical environments, studies on MMMs based on 2D MXenes have focused on liquid phase separations. Han et al. [153] reported a $2 \mathrm{D} \mathrm{Ti}_{3} \mathrm{C}_{2} \mathrm{~T}_{\mathrm{x}}$ ( $\mathrm{T}$ : functional groups, $\mathrm{O}, \mathrm{OH}$, and/or F) based mixed-matrix membrane and its application in water purification by forward osmosis. 2D MXene was prepared by etching and ultrasonic treatment on its parent $\mathrm{Ti}_{3} \mathrm{AlC}_{2}$ structure. The as-synthesized MXene nanosheets displayed a very loose lamellar structure, which provided a preferential transport channel for liquid molecules. It directly affected the water permeance of the fabricated MMM with polyethersulfone (PES). The PES/MXene membrane showed excellent water flux $\left(\sim 115 \mathrm{Lm}^{-2} \mathrm{~h}^{-1}\right)$ with high dye rejection $(\sim 92 \%$ for Congo Red with a molecular weight of 697). $\mathrm{Ti}_{3} \mathrm{C}_{2} \mathrm{~T}_{\mathrm{x}}$ nanosheet based mixed-matrix membranes were further tested in dehydration of conventional organic solvents. Submicron-Thick chitosan (Cs)/MXene mixed-matrix membrane was fabricated on porous polyacrylonitrile (PAN) substrate via a spin-coating method. The loose interlayer channels in the MXene provided a fast and selective transport pathway for water molecules. Their Cs/MXene mixed-matrix membrane showed a total flux of $1.4-1.5 \mathrm{~kg} \mathrm{~m}^{-2} \mathrm{~h}^{-1}$ and selectivity up to 1400,4800 , and 900 for dehydration of ethanol, ethyl acetate and dimethyl carbonate at $50^{\circ}$ C, respectively. Han et al. [154] also reported the MXene based cross-linked P84 copolyimide mixed-matrix membrane with improved solvent resistance. Organic solvent nanofiltration (OSN) was demonstrated using a triethylenetetramine (TETA) crosslinked P84/MXene mixed-matrix membrane. The water channel of MXene with good hydrophilicity played a positive role in the overall membrane performance. As a result, this membrane showed high flux $\left(268 \mathrm{Lm}^{-2} \mathrm{~h}^{-1}\right)$ and perfect rejection $(100 \%)$ to gentian violet with a molecular weight of 408. Moreover, the separation performance did not change during 18 days of immersion in acetone, methanol, and DMF, respectively.

\section{Final comments}

Rigid, microporous materials and membranes are emerging as a viable class of materials for energy-efficient separation processes. The combination of molecular specificity and chemical resistance put them to meet the challenges inherent in these types of difficult molecular separations. 2D materials like graphene and graphene oxide have already been shown to be successfully fabricated in membrane architecture to separate a number of different gas and liquid mixtures, and there is a rapid growth of sorption and transport data available for these materials that are helping to guide the design of new 2D materials such as MXene, $\mathrm{BN}, \mathrm{MoS}_{2}$, and 2D MOFs. However, much of the permeation data through the 2D-enabled membranes is still being gathered under different testing conditions that are not directly translatable to separation performance found in conventional polymeric membranes. Critically, the majority of these $2 \mathrm{D}$ materials have not yet been explored in various separation applications, therefore further research input is needed in this area.

We believe that future biofuel production, biorefining, and pharmaceutical separation will continue to integrate more membrane processes into its conventional processes. In pharmaceutical separation process, the highly selective membrane material is needed to remove either APIs (Active Pharmaceutical Ingredients), impurities, or to recover high-value catalysts utilized during synthesis. Optimized reuse of spent organic solvent is also achievable by ultrathin 2D-enabled membranes, where high solvent permeability and resistance required. A key asset 
of the 2D-enabled membrane is its high permeability (or permeance) that is directly translated into high-productivity of separation processes. Therefore, 2D-enabled membranes are expected to show promising applications in the field that requires organic solvent separations. However, long-term permeation stability and relative robustness of the $2 \mathrm{D}$-enabled membranes should be fully understood for these applications. Biofuel production and biorefining require energy-efficient removal of oxygenate species from various complex feeds. In this regards, 2D-enabled membranes with the hydrophobic surface are required to reduce the energy cost associated with the pervaporative recovery of bioalcohols from fermentation broths. Especially, membrane biofouling from the feed becomes an important issue to ensure the performance lifetime of the membranes. Hence, there is a critical need for fouling-related studies on 2D membranes that focusing on the interaction between biomolecules and different 2D nanosheets since the fouling mechanism on these membranes are relatively unexplored. Although the petrochemical industry has been implementing membrane processes, with more robust and selective membranes further low-energy petrochemical platform can be achieved. Since many of the 2D nanosheets are derived (exfoliated) from its parent chemically-resistant 3D frameworks, these nanosheets are also "solvent-ready" that will find vast applications where chemical resistance is required. Control over the swelling degree is essential to maintain stable separation performance over an extended time period [155]. These chemically and mechanically robust materials will withstand the harsh chemical conditions required to achieve liquid phase hydrocarbon separations. With refined selectivity, 2D-enabled membranes can further be used in reverse osmosis molecular differentiation of organic solvents that have been demonstrated with carbon molecular sieve hollow fiber membranes [156]. Furthermore, since the different 2D building blocks that have different molecular weight cutoffs, membrane cascade systems could be used to successively fractionate complex feeds.

Scalability and quality of the 2D membranes are also critical requirements for industrial applications. Class 1 and 2 membranes can potentially be processed via roll-to-roll production technique on porous flat support or even onto the hollow fiber support. Whereas class 3 membranes at this stage of development are deemed less-scalable. One important issue with class $3 \mathrm{mem}$ brane is that CVD grown materials still suffers from intrinsic defects, grain boundaries, and wrinkles that contribute to defective pathways. Even though with very small pinhole defects can compromise membrane performance in selectivity. Atomic-level surface contamination and the mechanism of the pinhole defect formation during synthesis should further be understood. While the majority of 2D-enabled membrane research has focused on developing new membrane materials, this is insufficient to transition these materials from lab scale to commercialization. One important issue is testing 2D-enabled membranes with more realistic feed mixtures. A membrane's pure component behavior is often not representative of its mixed component performance due to non-ideal component interactions. The development of user testbeds or dedicated user facilities for 2D-enabled membrane prototype testing would also be beneficial for the membrane-based separation areas. This would allow for more rigorous and uniform testing of promising new materials and reduce the cost. In addition to improved testing standards, a diverse portfolio of membranes will be needed to tackle the separation challenges of the future. For 2D-enabled membranes, varied approahces to membrane research and development will be needed to ensure the success of the membrane separations field. We believe these 2D-enabled membranes will continue to revolutionize the field of membrane-based separations by overcoming the limitations posed by conventional materials.

\section{Abbreviations}

ACF: Acid fuchsin; APIs: Active Pharmaceutical Ingredients; BDBA: 1,4benzenediboronic acid; BDC: 1,4-benzenedicarboxylate; BN: Boron Nitride; BPEl: Branched polyethyleneimine; BSA: Bovine serum albumin; COF: Covalent-organic framework; CR: Congo Red dye; Cs: Chitosan; CVD: Chemical-vapor deposition; dabco: 1.4-diazabicyclo[2.2.2] octane; EB: Ethidium bromide; EB: Evans blue; EtOH: Ethanol; FO: Forward osmosis; GO: Graphene oxide; h-BN: Hexagonal boron nitride; HPEl: Hyperbranched polyethyleneimine; LB: Langmuir-Blodgett; LbL: Layer-by-layer; LDH: Layered double hydroxide; MB: Methylene blue; $\mathrm{MeOH}$ : Methanol; MMM: Mixedmatrix membrane; MMT: Montmorillonite; MOF: Metal-organic framework; MoS2: Molybdenum Disulfide; MPD: m-phenylenediamine; MSF: Multi-stage flash; MWCO: Molecular weight cutoff; MWRO: Molecular weight retention onset; ndc: 1,4-naphthalenedicarboxylate; NF: Nanofiltration; OSN: Organic solvent nanofiltration; Pa: p-phenylenediamine; PAAH: Poly(allylamine hydrochloride); PAN: Polyacrylonitrile; PBI: Polybenzimidazole;

PDDA: Poly(diallyldimethylammonium chloride); PDMS: Polydimethylsiloxane; PEBA, Pebax ${ }^{\oplus}$ : Polyether-block-amide; PEG-PEI-GO: Polyethyleneiminefunctionalized GO sheets; PEl: Polyethyleneimine; PES: Polyethersulfone; PIMs: Polymers of intrinsic microporosity; PPA: Polypiperazine; PS: Polysulfone; PVA: Polyvinyl alcohol; PVAm: Polyvinylamine; PVDF: Polyvinylidene fluoride; R6G: Rhodamine 6G; rGO: Reduced graphene oxide; RhB: Rhodamine B; RO: Reverse osmosis; S-rGO: Solvated reduced graphene oxide; TD: 4,4'-pPhenylenedianiline; TETA: Triethylenetetramine; TFC: Thin film composite; TMC: Trimesol chloride; TMD: Transition metal dichalcogenide; Tp: 1,3,5triformylphloroglucinol; ZIF: Zeolitic-imidazole framework

\section{Acknowledgments}

This research was supported by Basic Science Research Program through the National Research Foundation of Korea (NRF) funded by the Ministry of Science, ICT \& Future Planning (NRF-2018R1C1B6002461). Also, this work was supported by the "International Collaborative Energy Technology R\&D Program" of the Korea Institute of Energy Technology Evaluation and Planning (KETEP) granted financial resource from the Ministry of Trade, Industry \& Energy, Republic of Korea (No. 20188550000580).

\section{Funding}

This research was supported by Basic Science Research Program through the National Research Foundation of Korea (NRF) funded by the Ministry of Science, ICT \& Future Planning (NRF-2018R1C1B6002461). Also, this work was 
supported by the "International Collaborative Energy Technology R\&D Program" of the Korea Institute of Energy Technology Evaluation and Planning (KETEP) granted financial resource from the Ministry of Trade, Industry \& Energy, Republic of Korea (No. 20188550000580).

\section{Availability of data and materials}

Not applicable.

\section{Authors' contributions}

DK designed and coordinated the research and drafted the manuscript. HT participated in the analysis of the emerging 2D materials and classification of 2D membranes and drafted the manuscript. JJ participated in the analysis of nanocomposites fabricated with 2D materials and drafted the manuscript. $A C$ and YKK participated in the analysis of surface chemistry and modification of 2D materials and drafted the manuscript. All authors read and approved the final manuscript.

\section{Competing interests}

The authors declare that they have no competing interests.

\section{Publisher's Note}

Springer Nature remains neutral with regard to jurisdictional claims in published maps and institutional affiliations.

\section{Author details}

'Department of Chemical and Biomolecular Engineering (BK-21 Plus), Korea Advanced Institute of Science and Technology (KAIST), Daejeon 34141, South Korea. ${ }^{2}$ Carbon Composite Materials Research Center, Institute of Advanced Composites Materials, Korea Institute of Science and Technology, Wanju-gun, Jeollabuk-do 565-905, South Korea. ${ }^{3}$ KAIST Institute for NanoCentury, Daejeon 34141, South Korea.

\section{Received: 3 January 2019 Accepted: 6 March 2019}

\section{Published online: 22 May 2019}

\section{References}

1. Sholl DS, Lively RP. Seven chemical separations: to change the world: purifying mixtures without using heat would lower global energy use, emissions and pollution--and open up new routes to resources. Nature. 2016;532:435+

2. Greenlee LF, Lawler DF, Freeman BD, Marrot B, Moulin P. Reverse osmosis desalination: water sources, technology, and today's challenges. Water Res 2009:43:2317-48.

3. Koros WJ, Lively RP. Water and beyond: expanding the spectrum of largescale energy efficient separation processes. AICHE J. 2012;58:2624-33.

4. Ulbricht M. Advanced functional polymer membranes. Polymer. 2006;47: 2217-62.

5. Karan S, Jiang Z, Livingston AG. Sub-10 nm polyamide nanofilms with ultrafast solvent transport for molecular separation. Science. 2015;348:134751.

6. Ma X, Kumar P, Mittal N, Khlyustova A, Daoutidis P, Mkhoyan KA, et al. Zeolitic imidazolate framework membranes made by ligand-induced permselectivation. Science. 2018;361:1008-11.

7. Chowdhury MR, Steffes J, Huey BD, McCutcheon JR. 3D printed polyamide membranes for desalination. Science. 2018;361:682-6.

8. Jue ML, Koh D-Y, McCool BA, Lively RP. Enabling widespread use of microporous materials for challenging organic solvent separations. Chem Mater. 2017;29:9863-76.

9. Thommes M, Kaneko K, Neimark AV, Olivier JP, Rodriguez-Reinoso F, Rouquerol J, et al. Physisorption of gases, with special reference to the evaluation of surface area and pore size distribution (IUPAC technical report). Pure Appl Chem. 2015;87:1051-69.

10. Nair RR, Wu HA, Jayaram PN, Grigorieva IV, Geim AK. Unimpeded permeation of water through helium-leak-tight graphene-based membranes. Science. 2012;335:442-4.

11. Kim HW, Yoon HW, Yoon S, Yoo BM, Ahn BK, Cho YH, et al. Selective gas transport through few-layered graphene and graphene oxide membranes. Science. 2013:342:91-6.

12. Huang K, Liu G, Lou Y, Dong Z, Shen J, Jin W. A graphene oxide membrane with highly selective molecular separation of aqueous organic solution. Angew Chem Int Ed. 2014;53:6929-32.
13. Akbari A, Sheath P, Martin ST, Shinde DB, Shaibani M, Banerjee PC, et al. Largearea graphene-based nanofiltration membranes by shear alignment of discotic nematic liquid crystals of graphene oxide. Nat Commun. 2016;7:1-12.

14. Huang L, Huang S, Venna SR, Lin H. Rightsizing Nanochannels in reduced graphene oxide membranes by solvating for dye desalination. Environ Sci Technol. 2018:52:12649-55.

15. Ibrahim A, Lin YS. Gas permeation and separation properties of large-sheet stacked graphene oxide membranes. J Memb Sci. 2018:550:238-45.

16. Guan K, Shen J, Liu G, Zhao J, Zhou H, Jin W. Spray-evaporation assembled graphene oxide membranes for selective hydrogen transport. Sep Purif Technol. 2017:174:126-35.

17. Mansouri J, Yapit E, Chen V. Polysulfone filtration membranes with isoporous structures prepared by a combination of dip-coating and breath figure approach. J Memb Sci. 2013;444:237-51.

18. Roth WJ, Nachtigall P, Morris RE, Jir C. Two-dimensional zeolites current status and perspectives. Chem Rev. 2014;114:4807-37.

19. Li Y, Yang W. Molecular sieve membranes: from 3D zeolites to 2D MOFs. Chinese J Catal. 2015;36:692-7.

20. Khayum MA, Kandambeth S, Mitra S, Nair SB, Das A, Nagane SS, et al. Chemically delaminated free-standing ultrathin covalent organic Nanosheets. Angew Chem Int Ed. 2016;55:15604-8.

21. Zhang H, Xiao Q, Guo X, Li N, Kumar P, Rangnekar N, et al. Open-pore twodimensional MFI zeolite nanosheets for the fabrication of hydrocarbonisomer-selective membranes on porous polymer supports. Angew Chem Int Ed. 2016;55:7184-7.

22. Jeon MY, Kim D, Kumar P, Lee PS, Rangnekar N, Bai P, et al. Ultra-selective high-flux membranes from directly synthesized zeolite nanosheets. Nature. 2017:543:690-4

23. Chen H, Wydra J, Zhang X, Lee P-S, Wang Z, Fan W, et al. Hydrothermal synthesis of zeolites with three-dimensionally ordered mesoporousimprinted structure. J Am Chem Soc. 2011;133:12390-3.

24. Kim D, Jeon MY, Stottrup BL, Tsapatsis M. Para-xylene ultra-selective zeolite MFI membranes fabricated from nanosheet monolayers at the air-water interface. Angew Chem Int Ed. 2018:57:480-5.

25. Graphene opens up to new applications. Nat Nanotechnol. 2015;10:381. https://www.nature.com/articles/nnano.2015.110\#article-info.

26. Xu WL, Zhou F, Yu M. Tuning water nanofiltration performance of fewlayered, reduced graphene oxide membranes by oxygen plasma. Ind Eng Chem Res. 2018;57:16103-9.

27. Peng $Y$, Li Y, Ban Y, Yang W. Two-dimensional metal-organic framework Nanosheets for membrane-based gas separation. Angew Chem Int Ed. 2017; 56:9757-61.

28. Koh D-Y, Lively RP. Membranes at the limit. Nat Nanotechnol. 2015;10:385

29. Surwade SP, Smirnov SN, Vlassiouk IV, Unocic RR, Veith GM, Dai S, et al. Water desalination using nanoporous single-layer graphene. Nat Nanotechnol. 2015;10:459-64.

30. Huang S, Dakhchoune M, Luo W, Oveisi E, He G, Rezaei M, et al. Single-layer graphene membranes by crack-free transfer for gas mixture separation. Nat Commun. 2018:9:2632.

31. Agrawal KV, Benck JD, Yuan Z, Misra RP, Govind Rajan A, Eatmon Y, et al. Fabrication, pressure testing, and Nanopore formation of single-layer graphene membranes. J Phys Chem C. 2017;121:14312-21.

32. Yuan Z, Govind Rajan A, Misra RP, Drahushuk LW, Agrawal KV, Strano MS, et al. Mechanism and prediction of gas permeation through sub-nanometer graphene pores: comparison of theory and simulation. ACS Nano. 2017:11: 7974-87.

33. Liu H, Dai S, Jiang D. Permeance of $\mathrm{H} 2$ through porous graphene from molecular dynamics. Solid State Commun. 2013;175(176):101-5.

34. Drahushuk LW, Strano MS. Mechanisms of gas permeation through single layer graphene membranes. Langmuir. 2012;28:16671-8.

35. Wang L, Boutilier MSH, Kidambi PR, Jang D, Hadjiconstantinou NG, Karnik R. Fundamental transport mechanisms, fabrication and potential applications of nanoporous atomically thin membranes. Nat Nanotechnol. 2017;12:509.

36. Tao Y, Xue Q, Liu Z, Shan M, Ling C, Wu T, et al. Tunable hydrogen separation in porous graphene membrane: first-principle and molecular dynamic simulation. ACS Appl Mater Interfaces. 2014;6:8048-58.

37. Du H, Li J, Zhang J, Su G, Li X, Zhao Y. Separation of hydrogen and nitrogen gases with porous graphene membrane. J Phys Chem C. 2011;115:23261-6.

38. Sun C, Boutilier MSH, Au H, Poesio P, Bai B, Karnik R, et al. Mechanisms of molecular permeation through Nanoporous graphene membranes. Langmuir. 2014;30:675-82. 
39. Park HB, Kamcev J, Robeson LM, Elimelech M, Freeman BD. Maximizing the right stuff: the trade-off between membrane permeability and selectivity. Science (80- ). 2017;356:eaab0530.

40. Lei W, Portehault D, Liu D, Qin S, Chen Y. Porous boron nitride nanosheets for effective water cleaning. Nat Commun. 2013;4:1777.

41. Naguib M, Mochalin VN, Barsoum MW, Gogotsi Y. 25th anniversary article: MXenes: a new family of two-dimensional materials. Adv Mater. 2014;26: 992-1005.

42. Ding $L$, Wei $Y$, Li L, Zhang T, Wang H, Xue J, et al. MXene molecular sieving membranes for highly efficient gas separation. Nat Commun. 2018:9:155

43. Shen J, Liu G, Ji Y, Liu Q, Cheng L, Guan K, et al. 2D MXene Nanofilms with tunable gas transport channels. Adv Funct Mater. 2018;28:1801511.

44. Ren CE, Hatzell KB, Alhabeb M, Ling Z, Mahmoud KA, Gogotsi Y. Chargeand size-selective ion sieving through Ti3C2Tx MXene membranes. J Phys Chem Lett. 2015:6:4026-31.

45. Wang J, Chen P, Shi B, Guo W, Jaroniec M, Qiao S-Z. A regularly channeled lamellar membrane for unparalleled water and organics permeation. Angew Chem Int Ed. 2018;57:6814-8

46. Li L, Zhang T, Duan Y, Wei Y, Dong C, Ding L, et al. Selective gas diffusion in two-dimensional MXene lamellar membranes: insights from molecular dynamics simulations. J Mater Chem A. 2018:6:11734-42.

47. Song L, Ci L, Lu H, Sorokin PB, Jin C, Ni J, et al. Large scale growth and characterization of atomic hexagonal boron nitride layers. Nano Lett. 2010; 10:3209-15.

48. Chen Y, Zou J, Campbell SJ, Le Caer G. Boron nitride nanotubes: Pronounced resistance to oxidation. Appl Phys Lett. 2004;84:2430-2.

49. Golberg D, Bando Y, Huang Y, Terao T, Mitome M, Tang C, et al. Boron nitride nanotubes and Nanosheets. ACS Nano. 2010:4:2979-93.

50. Qin S, Liu D, Wang G, Portehault D, Garvey CJ, Gogotsi Y, et al. High and stable ionic conductivity in 2D Nanofluidic ion channels between boron nitride layers. J Am Chem Soc. 2017;139:6314-20.

51. Kostoglou N, Polychronopoulou K, Rebholz C. Thermal and chemical stability of hexagonal boron nitride (h-BN) nanoplatelets. Vacuum. 2015;112:42-5.

52. Lin Y, Connell JW. Advances in $2 \mathrm{D}$ boron nitride nanostructures: nanosheets, nanoribbons, nanomeshes, and hybrids with graphene. Nanoscale. 2012:4:6908-39.

53. Sutter $P$, Lahiri J, Albrecht $P$, Sutter E. Chemical vapor deposition and etching of high-quality monolayer hexagonal boron nitride films. ACS Nano. 2011:5:7303-9.

54. Chen C, Wang J, Liu D, Yang C, Liu Y, Ruoff RS, et al. Functionalized boron nitride membranes with ultrafast solvent transport performance for molecular separation. Nat Commun. 2018;9:1902.

55. Liu D, He L, Lei W, Klika KD, Kong L, Chen Y. Multifunctional polymer/porous boron nitride Nanosheet membranes for superior trapping emulsified oils and organic molecules. Adv Mater Interfaces. 2015;2:1500228.

56. Deng M, Kwac K, Li M, Jung Y, Park HG. Stability, molecular sieving, and ion diffusion selectivity of a lamellar membrane from two-dimensional molybdenum disulfide. Nano Lett. 2017;17:2342-8.

57. Tan C, Cao X, Wu XJ, He Q, Yang J, Zhang X, et al. Recent advances in ultrathin two-dimensional nanomaterials. Chem Rev. 2017;117:6225-331.

58. Voiry D, Mohite A, Chhowalla M. Phase engineering of transition meta dichalcogenides. Chem Soc Rev. 2015;44:2702-12.

59. Hirunpinyopas W, Prestat E, Worrall SD, Haigh SJ, Dryfe RAW, Bissett MA. Desalination and nanofiltration through functionalized laminar MoS2 membranes. ACS Nano. 2017:11:11082-90.

60. Heiranian M, Farimani AB, Aluru NR. Water desalination with a single-layer MoS2 nanopore. Nat Commun. 2015;6:8616.

61. Wang Z, Tu Q, Zheng S, Urban JJ, Li S, Mi B. Understanding the aqueous stability and filtration capability of MoS2 membranes. Nano Lett. 2017;17: 7289-98.

62. Chen $D$, Ying $W$, Guo $Y$, Ying $Y$, Peng $X$. Enhanced gas separation through Nanoconfined ionic liquid in laminated MoS2 membrane. ACS Appl Mater Interfaces. 2017:9:44251-7.

63. Li M-N, Sun X-F, Wang L, Wang S-Y, Afzal MZ, Song C, et al. Forward osmosis membranes modified with laminar MoS2 nanosheet to improve desalination performance and antifouling properties. Desalination. 2018:436:107-13.

64. Moghadam PZ, Li A, Wiggin SB, Tao A, Maloney AGP, Wood PA, et al. Development of a Cambridge structural database subset: a collection of metalorganic frameworks for past, present, and future. Chem Mater. 2017;29:2618-25.

65. Gomez DA, Toda J, Sastre G. Screening of hypothetical metal-organic frameworks for H2 storage. Phys Chem Chem Phys. 2014;16:19001-10.
66. Yan $R$, Zhao $Y$, Yang $H$, Kang X-J, Wang C, Wen L-L, et al. Ultrasmall au nanoparticles embedded in 2D mixed-ligand metal-organic framework Nanosheets exhibiting highly efficient and size-selective catalysis. Adv Funct Mater. 2018;28:1802021.

67. Li Y-H, Wang S-L, Su Y-C, Ko B-T, Tsai C-Y, Lin C-H. Microporous 2D indium metal-organic frameworks for selective $\mathrm{CO} 2$ capture and their application in the catalytic CO2-cycloaddition of epoxides. Dalt Trans. 2018;47:9474-81.

68. Kondo A, Noguchi H, Carlucci L, Proserpio DM, Ciani G, Kajiro H, et al. Double-step gas sorption of a two-dimensional metal-organic framework. J Am Chem Soc. 2007;129:12362-3.

69. Zhai F, Zheng Q, Chen Z, Ling Y, Liu X, Weng L, et al. Crystal transformation synthesis of a highly stable phosphonate MOF for selective adsorption of CO2. CrystEngComm. 2013;15:2040-3.

70. Hu X-L, Liu F-H, Qin C, Shao K-Z, Su Z-M. A 2D bilayered metal-organic framework as a fluorescent sensor for highly selective sensing of nitro explosives. Dalt Trans. 2015;44:7822-7.

71. Rachuri Y, Parmar B, Bisht KK, Suresh E. Mixed ligand two dimensional Cd (ii)/Ni (ii) metal organic frameworks containing dicarboxylate and tripodal $\mathrm{N}$ donor ligands: Cd (ii) MOF is an efficient luminescent sensor for detection of picric acid in aqueous media. Dalt Trans. 2016:45:7881-92.

72. Xu G, Yamada T, Otsubo K, Sakaida S, Kitagawa H. Facile "modular assembly" for fast construction of a highly oriented crystalline MOF Nanofilm. J Am Chem Soc. 2012;134:16524-7.

73. Abhervé A, Mañas-Valero S, Clemente-León M, Coronado E. Graphene related magnetic materials: micromechanical exfoliation of 2D layered magnets based on bimetallic anilate complexes with inserted [Felll (acac2trien)]+ and [Felll (sal2-trien)]+ molecules. Chem Sci. 2015;6:4665-73.

74. Ashworth DJ, Foster JA. Metal-organic framework nanosheets (MONs): a new dimension in materials chemistry. J Mater Chem A. 2018;6:16292-307.

75. Yuan $Y$, Wang W, Qiu L, Peng F, Jiang X, Xie A, et al. Surfactant-assisted facile synthesis of fluorescent zinc benzenedicarboxylate metal-organic framework nanorods with enhanced nitrobenzene explosives detection. Mater Chem Phys. 2011;131:358-61

76. Zhao M, Wang Y, Ma Q, Huang Y, Zhang X, Ping J, et al. Ultrathin 2D metalorganic framework Nanosheets. Adv Mater. 2015;27:7372-8.

77. Pham M-H, Vuong G-T, Fontaine F-G, Do T-O. Rational synthesis of metalorganic framework Nanocubes and Nanosheets using selective modulators and their morphology-dependent gas-sorption properties. Cryst Growth Des. 2012;12:3091-5.

78. Cao F, Zhao M, Yu Y, Chen B, Huang Y, Yang J, et al. Synthesis of twodimensional CoS1.097/nitrogen-doped carbon nanocomposites using metal-organic framework Nanosheets as precursors for supercapacitor application. J Am Chem Soc. 2016;138:6924-7.

79. Novoselov KS, Geim AK, Morozov SV, Jiang D, Zhang Y, Dubonos SV, et al. Electric field effect in atomically thin carbon films. Science. 2004;306:666-9.

80. Peng $Y$, Li Y, Ban Y, Jin H, Jiao W, Liu X, et al. Metal-organic framework nanosheets as building blocks for molecular sieving membranes. Science. 2014;346:1356-9.

81. Cliffe MJ, Castillo-Martínez E, Wu Y, Lee J, Forse AC, Firth FCN, et al. Metalorganic Nanosheets formed via defect-mediated transformation of a hafnium metal-organic framework. J Am Chem Soc. 2017;139:5397-404.

82. Xu M, Yuan S, Chen X-Y, Chang Y-J, Day G, Gu Z-Y, et al. Two-dimensional metal-organic framework Nanosheets as an enzyme inhibitor: modulation of the a-chymotrypsin activity. J Am Chem Soc. 2017;139:8312-9.

83. Backes C, Higgins TM, Kelly A, Boland C, Harvey A, Hanlon D, et al. Guidelines for exfoliation, characterization and processing of layered materials produced by liquid exfoliation. Chem Mater. 2017;29:243-55.

84. Wang X, Chi C, Zhang K, Qian Y, Gupta KM, Kang Z, et al. Reversed thermoswitchable molecular sieving membranes composed of two-dimensional metal-organic nanosheets for gas separation. Nat Commun. 2017;8:1-10

85. Kandambeth S, Biswal BP, Chaudhari HD, Rout KC, Kunjattu HS, Mitra S, et al. Selective molecular sieving in self-standing porous covalent-organicframework membranes. Adv Mater. 2017;29:1-9.

86. Zhang W, Zhang L, Zhao H, Li B, Ma H. A two-dimensional cationic covalent organic framework membrane for selective molecular sieving. J Mater Chem A. 2018:6:13331-9.

87. Shinde DB, Sheng G, Li X, Ostwal M, Emwas A-H, Huang K-W, et al. Crystalline 2D covalent organic framework membranes for high-flux organic solvent Nanofiltration. J Am Chem Soc. 2018;140:14342-9. 
88. Gadwal I, Sheng G, Thankamony RL, Liu Y, Li H, Lai Z. Synthesis of Sub-10 $\mathrm{nm}$ two-dimensional covalent organic thin film with sharp molecular sieving Nanofiltration. ACS Appl Mater Interfaces. 2018;10:12295-9.

89. Zhang K, He Z, Gupta KM, Jiang J. Computational design of 2D functional covalent-organic framework membranes for water desalination. Environ Sci Water Res Technol. 2017;3:735-43.

90. Li G, Zhang K, Tsuru T. Two-dimensional covalent organic framework (COF) membranes fabricated via the assembly of exfoliated COF Nanosheets. ACS Appl Mater Interfaces. 2017;9:8433-6.

91. Tong M, Yang Q, Ma Q, Liu D, Zhong C. Few-layered ultrathin covalent organic framework membranes for gas separation: a computational study. J Mater Chem A. 2016:4:124-31.

92. Hegab HM, Zou L. Graphene oxide-assisted membranes: fabrication and potential applications in desalination and water purification. J Memb Sci. 2015:484:95-106.

93. Hong T-K, Lee DW, Choi HJ, Shin HS, Kim B-S. Transparent, flexible conducting hybrid multilayer thin films of multiwalled carbon nanotubes with graphene Nanosheets. ACS Nano. 2010;4:3861-8.

94. Bourlinos AB, Gournis D, Petridis D, Szabó T, Szeri A, Dékány I. Graphite oxide: chemical reduction to graphite and surface modification with primary aliphatic amines and amino acids. Langmuir. 2003;19:6050-5.

95. Cheng HKF, Sahoo NG, Tan YP, Pan Y, Bao H, Li L, et al. Poly (vinyl alcohol) nanocomposites filled with poly (vinyl alcohol)-grafted graphene oxide. ACS Appl Mater Interfaces. 2012;4:2387-94.

96. Matsuo Y, Fukunaga T, Fukutsuka T, Sugie Y. Silylation of graphite oxide; 2004. https://doi.org/10.1016/j.carbon.2004.03.024.

97. Choi W, Choi J, Bang J, Lee J-H. Layer-by-layer assembly of graphene oxide Nanosheets on polyamide membranes for durable reverse-osmosis applications. ACS Appl Mater Interfaces. 2013;5:12510-9.

98. Nam YT, Choi J, Kang KM, Kim DW, Jung H-T. Enhanced stability of laminated graphene oxide membranes for Nanofiltration via interstitial amide bonding. ACS Appl Mater Interfaces. 2016;8:27376-82.

99. Yuan Y, Gao X, Wei Y, Wang X, Wang J, Zhang Y, et al. Enhanced desalination performance of carboxyl functionalized graphene oxide nanofiltration membranes. Desalination. 2017;405:29-39.

100. Xu Z, Zhang J, Shan M, Li Y, Li B, Niu J, et al. Organosilane-functionalized graphene oxide for enhanced antifouling and mechanical properties of polyvinylidene fluoride ultrafiltration membranes. J Memb Sci. 2014;458:1-13.

101. Lee B, Li K, Yoon HS, Yoon J, Mok Y, Lee Y, et al. Membrane of functionalized reduced graphene oxide Nanoplates with angstrom-level channels. Sci Rep. 2016;6:28052.

102. Hu M, Mi B. Layer-by-layer assembly of graphene oxide membranes via electrostatic interaction. J Memb Sci. 2014:469:80-7.

103. Zhao J, Zhu Y, Pan F, He G, Fang C, Cao K, et al. Fabricating graphene oxide-based ultrathin hybrid membrane for pervaporation dehydration via layer-by-layer self-assembly driven by multiple interactions. J Memb Sci. 2015:487:162-72.

104. Lian B, Deng J, Leslie G, Bustamante H, Sahajwalla V, Nishina Y, et al. Surfactant modified graphene oxide laminates for filtration. Carbon. 2017; 116:240-5.

105. Sun L, Huang H, Peng X. Laminar MoS2 membranes for molecule separation. Chem Commun. 2013;49:10718-20.

106. Presolski S, Pumera M. Covalent functionalization of MoS2. Mater Today. 2016;19:140-5

107. Chou SS, De M, Kim J, Byun S, Dykstra C, Yu J, et al. Ligand conjugation of chemically exfoliated MoS2. J Am Chem Soc. 2013;135:4584-7.

108. Zhou L, He B, Yang Y, He Y. Facile approach to surface functionalized MoS2 nanosheets. RSC Adv. 2014;4:32570-8.

109. Zhou K, Gao R, Gui Z, Hu Y. The effective reinforcements of functionalized MoS2 nanosheets in polymer hybrid composites by sol-gel technique. Compos Part A Appl Sci Manuf. 2017;94:1-9.

110. Zhou K, Jiang S, Shi Y, Liu J, Wang B, Hu Y, et al. Multigram-scale fabrication of organic modified MoS2 nanosheets dispersed in polystyrene with improved thermal stability, fire resistance, and smoke suppression properties. RSC Adv. 2014;4:40170-80.

111. Qin S, Liu D, Chen Y, Chen C, Wang G, Wang J, et al. Nanofluidic electric generators constructed from boron nitride nanosheet membranes. Nano Energy. 2018;47:368-73.

112. Li Z, Zhang Y, Chan C, Zhi C, Cheng X, Fan J. Temperature-dependent lipid extraction from membranes by boron nitride Nanosheets. ACS Nano. 2018; 12:2764-72
113. Weber M, Koonkaew B, Balme S, Utke I, Picaud F, latsunskyi I, et al. Boron nitride Nanoporous membranes with high surface charge by atomic layer deposition. ACS Appl Mater Interfaces. 2017;9:16669-78.

114. Lin Y, Williams TV, Xu T-B, Cao W, Elsayed-Ali HE, Connell JW. Aqueous dispersions of few-layered and Monolayered hexagonal boron nitride Nanosheets from sonication-assisted hydrolysis: critical role of water. J Phys Chem C. 2011;115:2679-85.

115. Kim D, Muramatsu H, Kim YA. Hydrolytic unzipping of boron nitride nanotubes in nitric acid. Nanoscale Res Lett. 2017;12:94.

116. Du M, Wu Y, Hao X. A facile chemical exfoliation method to obtain large size boron nitride nanosheets. CrystEngComm. 2013;15:1782-6.

117. Sainsbury T, Satti A, May P, Wang Z, McGovern I, Gun'ko YK, et al. Oxygen radical functionalization of boron nitride Nanosheets. J Am Chem Soc. 2012 134:18758-71.

118. Li X, Hao X, Zhao M, Wu Y, Yang J, Tian Y, et al. Exfoliation of hexagonal boron nitride by molten hydroxides. Adv Mater. 2013;25:2200-4.

119. Nie R, Sang R, Ma X, Zheng Y, Cheng X, Li W, et al. Copper- $\gamma$-cyclodextrin complexes immobilized on hexagonal boron nitride as an efficient catalyst in the multicomponent synthesis of 1,2,3-triazoles. J Catal. 2016;344:286-92.

120. Hou J, Li G, Yang N, Qin L, Grami ME, Zhang Q, et al. Preparation and characterization of surface modified boron nitride epoxy composites with enhanced thermal conductivity. RSC Adv. 2014;4:44282-90.

121. Lei W, Mochalin VN, Liu D, Qin S, Gogotsi Y, Chen Y. Boron nitride colloidal solutions, ultralight aerogels and freestanding membranes through onestep exfoliation and functionalization. Nat Commun. 2015;6:8849.

122. Abdikheibari S, Lei W, Dumée LF, Milne N, Baskaran K. Thin film nanocomposite nanofiltration membranes from amine functionalized-boron nitride/polypiperazine amide with enhanced flux and fouling resistance. J Mater Chem A. 2018;6:12066-81.

123. Zhao J, Yang Y, Yang C, Tian Y, Han Y, Liu J, et al. A hydrophobic surface enabled salt-blocking 2D Ti3C2 MXene membrane for efficient and stable solar desalination. J Mater Chem A. 2018;6:16196-204.

124. Liu G, Shen J, Liu Q, Liu G, Xiong J, Yang J, et al. Ultrathin two-dimensional MXene membrane for pervaporation desalination. J Memb Sci. 2018:548: 548-58.

125. Rasool K, Mahmoud KA, Johnson DJ, Helal M, Berdiyorov GR, Gogotsi Y. Efficient antibacterial membrane based on two-dimensional Ti3C2Tx (MXene) Nanosheets. Sci Rep. 2017;7:1598.

126. Naguib M, Kurtoglu M, Presser V, Lu J, Niu J, Heon M, et al. Twodimensional nanocrystals produced by exfoliation of Ti3AIC2. Adv Mater. 2011;23:4248-53.

127. Srivastava $P$, Mishra A, Mizuseki H, Lee K-R, Singh AK. Mechanistic insight into the chemical exfoliation and functionalization of Ti3C2 MXene. ACS Appl Mater Interfaces. 2016;8:24256-64.

128. Ghidiu M, Lukatskaya MR, Zhao M-Q, Gogotsi Y, Barsoum MW. Conductive two-dimensional titanium carbide 'clay' with high volumetric capacitance. Nature. 2014:516:78.

129. Wang H, Zhang J, Wu Y, Huang H, Li G, Zhang X, et al. Surface modified MXene Ti3C2 multilayers by aryl diazonium salts leading to large-scale delamination. Appl Surf Sci. 2016;384:287-93.

130. Wang $H$, Zhang J, Wu Y, Huang H, Jiang Q. Chemically functionalized twodimensional titanium carbide MXene by in situ grafting-intercalating with diazonium ions to enhance supercapacitive performance. J Phys Chem Solids. 2018;115:172-9.

131. Ling Z, Ren CE, Zhao M-Q, Yang J, Giammarco JM, Qiu J, et al. Flexible and conductive MXene films and nanocomposites with high capacitance. Proc Natl Acad Sci. 2014;111:16676-81.

132. Wu X, Hao L, Zhang J, Zhang X, Wang J, Liu J. Polymer-Ti3C2Tx composite membranes to overcome the trade-off in solvent resistant nanofiltration for alcohol-based system. J Memb Sci. 2016;515:175-88.

133. Ding $L$, Wei $Y$, Wang $Y$, Chen $H$, Caro J, Wang $H$. A two-dimensional lamellar membrane: MXene Nanosheet stacks. Angew Chem Int Ed. 2017;56:1825-9.

134. Park S-J, Ahn W-G, Choi W, Park S-H, Lee JS, Jung HW, et al. A facile and scalable fabrication method for thin film composite reverse osmosis membranes: dual-layer slot coating. J Mater Chem A. 2017;5:6648-55.

135. Sukitpaneenit $P$, Chung T-S. High performance thin-film composite forward osmosis hollow fiber membranes with macrovoid-free and highly porous structure for sustainable water production. Environ Sci Technol. 2012;46:7358-65.

136. Li Y, Yang S, Zhang K, Van der Bruggen B. Thin film nanocomposite reverse osmosis membrane modified by two dimensional laminar MoS2 with 
improved desalination performance and fouling-resistant characteristics. Desalination. 2019;454:48-58.

137. Dong H, Wu L, Zhang L, Chen H, Gao C. Clay nanosheets as charged filler materials for high-performance and fouling-resistant thin film nanocomposite membranes. J Memb Sci. 2015;494:92-103.

138. Shen J, Zhang M, Liu G, Guan K, Jin W. Size effects of graphene oxide on mixed matrix membranes for CO2 separation. AICHE J. 2016;62:2843-52.

139. Dong G, Hou J, Wang J, Zhang Y, Chen V, Liu J. Enhanced CO2/N2 separation by porous reduced graphene oxide/Pebax mixed matrix membranes. J Memb Sci. 2016;520:860-8.

140. Shen Y, Wang H, Liu J, Zhang Y. Enhanced performance of a novel polyvinyl amine/chitosan/graphene oxide mixed matrix membrane for $\mathrm{CO} 2$ capture. ACS Sustain Chem Eng. 2015;3:1819-29.

141. Li X, Cheng Y, Zhang H, Wang S, Jiang Z, Guo R, et al. Efficient CO2 capture by functionalized graphene oxide Nanosheets as fillers to fabricate multiPermselective mixed matrix membranes. ACS Appl Mater Interfaces. 2015;7: 5528-37.

142. Yin J, Zhu G, Deng B. Graphene oxide (GO) enhanced polyamide (PA) thinfilm nanocomposite (TFN) membrane for water purification. Desalination. 2016;379:93-101.

143. Ganesh BM, Isloor AM, Ismail AF. Enhanced hydrophilicity and salt rejection study of graphene oxide-polysulfone mixed matrix membrane. Desalination. 2013;313:199-207.

144. Yang D, Yang S, Jiang Z, Yu S, Zhang J, Pan F, et al. Polydimethyl siloxanegraphene nanosheets hybrid membranes with enhanced pervaporative desulfurization performance. J Memb Sci. 2015;487:152-61.

145. Li Y, Krantz WB, Chung T-S. A novel primer to prevent nanoparticle agglomeration in mixed matrix membranes. AICHE J. 2007;53:2470-5.

146. Moore $\Pi$, Koros WJ. Non-ideal effects in organic-inorganic materials for gas separation membranes. J Mol Struct. 2005;739:87-98.

147. Rodenas T, Luz I, Prieto G, Seoane B, Miro H, Corma A, et al. Metal-organic framework nanosheets in polymer composite materials for gas separation. Nat Mater. 2014;14:48.

148. Shete M, Kumar P, Bachman JE, Ma X, Smith ZP, Xu W, et al. On the direct synthesis of $\mathrm{Cu}(\mathrm{BDC}) \mathrm{MOF}$ nanosheets and their performance in mixed matrix membranes. J Memb Sci. 2018;549:312-20.

149. Kang Z, Peng Y, Hu Z, Qian Y, Chi C, Yeo LY, et al. Mixed matrix membranes composed of two-dimensional metal-organic framework nanosheets for pre-combustion CO2capture: a relationship study of filler morphology versus membrane performance. J Mater Chem A. 2015:3:20801-10.

150. Cheng Y, Wang X, Jia C, Wang Y, Zhai L, Wang $Q$, et al. Ultrathin mixed matrix membranes containing two-dimensional metal-organic framework nanosheets for efficient CO2/CH4separation. J Memb Sci. 2017;539:213-23.

151. Samarasinghe SASC, Chuah CY, Yang Y, Bae T-H. Tailoring CO2/CH4 separation properties of mixed-matrix membranes via combined use of two- and threedimensional metal-organic frameworks. J Memb Sci. 2018;557:30-7.

152. Maleski K, Mochalin VN, Gogotsi Y. Dispersions of two-dimensional titanium carbide MXene in organic solvents. Chem Mater. 2017;29:1632-40.

153. Han R, Ma X, Xie Y, Teng D, Zhang S. Preparation of a new 2D MXene/PES composite membrane with excellent hydrophilicity and high flux. RSC Adv. 2017;7:56204-10.

154. Han R, Xie Y, Ma X. Crosslinked P84 copolyimide/MXene mixed matrix membrane with excellent solvent resistance and permselectivity. Chinese J Chem Eng. 2018. https://doi.org/10.1016/j.cjche.2018.10.005

155. Eum K, Ma C, Koh D-Y, Rashidi F, Li Z, Jones CW, et al. Zeolitic Imidazolate framework membranes supported on macroporous carbon hollow fibers by fluidic processing techniques. Adv Mater Interfaces. 2017;4:1700080,

156. Koh D-Y, McCool BA, Deckman HW, Lively RP. Reverse osmosis molecular differentiation of organic liquids using carbon molecular sieve membranes. Science. 2016;353:804-7.

157. Li H, Song Z, Zhang X, Huang Y, Li S, Mao Y, et al. Ultrathin, molecularsieving graphene oxide membranes for selective hydrogen separation. Science. 2013;342:95-8.

158. Shen J, Liu G, Huang K, Chu Z, Jin W, Xu N. Subnanometer twodimensional graphene oxide channels for ultrafast gas sieving. ACS Nano. 2016;10:3398-409

159. Wang $D$, Wang Z, Wang L, Hu L, Jin J. Ultrathin membranes of singlelayered MoS2 nanosheets for high-permeance hydrogen separation. Nanoscale. 2015;7:17649-52.
160. Celebi K, Buchheim J, Wyss RM, Droudian A, Gasser P, Shorubalko I, et al. Ultimate permeation across atomically thin porous graphene. Science. 2014; 344:289-92

161. Kang Z, Peng Y, Qian Y, Yuan D, Addicoat MA, Heine T, et al. Mixed matrix membranes (MMMs) comprising exfoliated 2D covalent organic frameworks (COFs) for efficient CO2 separation. Chem Mater. 2016;28:1277-85.

162. Robeson LM. The upper bound revisited. J Memb Sci. 2008;320:390-400

163. Han B, Hu YH. MoS2 as a co-catalyst for photocatalytic hydrogen production from water. Energy Sci Eng. 2016;4:285-304.

164. Zeng Y, Zou R, Zhao Y. Covalent organic frameworks for CO2 capture. Adv Mater. 2016:28:2855-73.

\section{Ready to submit your research? Choose BMC and benefit from:}

- fast, convenient online submission

- thorough peer review by experienced researchers in your field

- rapid publication on acceptance

- support for research data, including large and complex data types

- gold Open Access which fosters wider collaboration and increased citations

- maximum visibility for your research: over $100 \mathrm{M}$ website views per year

At BMC, research is always in progress.

Learn more biomedcentral.com/submissions 